\title{
Plane-Wave Dynamics of Optical Parametric Oscillation with Simultaneous Sum-Frequency Generation
}

\author{
Yamaç Dikmelik, Student Member, IEEE, Gülbin Akgün, and Orhan Aytür, Member, IEEE
}

\begin{abstract}
This paper presents a theoretical analysis of sumfrequency generating optical parametric oscillators where a single nonlinear crystal is used for both parametric generation and sum-frequency generation. In these devices, the parametric and sum-frequency generation processes are both phase matched for the same direction of propagation inside the crystal. Different polarization geometries for which this simultaneous phase-matching condition can potentially be satisfied are identified and categorized, for both birefringent and quasi-phase-matching methods. Plane-wave coupled-mode equations are presented for each of these categories. Solutions of these coupled mode equations and calculation of the single-pass saturated signal gain are outlined. Intracavity signal photon flux density calculations based on these solutions lead to stable steady-state upconversion, multistability, and chaos. The dependence of the photon conversion efficiency on various design parameters are investigated.
\end{abstract}

Index Terms - Chaos, nonlinear frequency conversion, optical bistability, optical parametric oscillators, parametric devices, quasi-phase matching, sum-frequency generation.

\section{INTRODUCTION}

$\mathbf{O}$ PTICAL parametric oscillators (OPO's) are widely used for tunable wavelength conversion of lasers to previously unavailable wavelength ranges [1]-[3]. By itself, an OPO can only provide downconversion to longer wavelengths. Upconversion to shorter wavelengths is achieved with the use of a second nonlinear element for frequency doubling [4] or sum-frequency generation (SFG) [5]. This second nonlinear crystal is usually internal to the OPO cavity to take advantage of the high intracavity field intensities. The plane-wave theory of these two-crystal intracavity upconversion OPO's have been studied extensively [6]-[8].

Single-crystal upconversion OPO's, where frequency doubling [9] or SFG [10] takes place within the OPO crystal itself, have recently been demonstrated, providing new and highly efficient schemes for the frequency upconversion of lasers. These devices are based on the premise that optical parametric amplification and SFG or frequency doubling can be simultaneously phase matched inside the same nonlinear crystal. This simultaneous phase matching condition may be

Manuscript received July 20, 1998; revised January 5, 1999. This work was supported in part by the Turkish Scientific and Technical Research Council (Tubitak) under Grant 197E050.

The authors are with the Department of Electrical and Electronics Engineering, Bilkent University, TR-06533 Bilkent, Ankara, Turkey.

Publisher Item Identifier S 0018-9197(99)04107-X. achieved with either birefringent phase matching (BPM) or quasi-phase matching (QPM). We have identified a number of common nonlinear crystals and wavelengths where two second-order nonlinear processes are simultaneously phase matched with BPM. QPM techniques using periodically poled materials provide even larger numbers of possibilities and much more design flexibility.

The first demonstration of simultaneous phase matching of two different second-order interactions was reported in ammonium dihydrogen phosphate [11]. Shortly afterwards, three second-order interactions were reported to be simultaneously phase matched in lithium niobate [12]. Recently, there has been an increasing number of frequency conversion experiments that report the use of the simultaneous phasematching technique. Single-crystal upconversion OPO's that employ simultaneous BPM of frequency doubling [9] or SFG [10], in $\mathrm{KTiOPO}_{4}$ (KTP), and periodically poled lithium niobate (PPLN) OPO's with simultaneous higher order QPM of frequency doubling [13]-[15] or SFG [15], [16] have been reported. A cascaded OPO, where the signal of a primary OPO acts as the pump for a secondary OPO, has been demonstrated in PPLN with first-order QPM for both OPO processes [17]. Simultaneous SFG of the pump and the idler in a $\beta-\mathrm{BaB}_{2} \mathrm{O}_{4}$ (BBO) crystal optical parametric amplifier (OPA) has been achieved with BPM [18]. Third-harmonic generation in PPLN with simultaneous first-order QPM of the frequency doubling and SFG processes has also been demonstrated [19].

In simultaneously phase-matched frequency conversion devices, the interacting beams are governed by new sets of coupled-mode equations [20]. The steady-state plane-wave analysis of various single-crystal frequency doubling OPO's (or self-doubling OPO's) have yielded promising results in terms of conversion efficiency [20]. In this paper, we present a plane-wave analysis of simultaneous parametric oscillation and SFG, where the resonant OPO signal field is summed with the pump field. We first identify and classify all polarization geometries for which simultaneous phase matching may be achieved and present the associated sets of coupled-mode equations for each geometry. We proceed with a discussion of the single-pass solutions of these equations, followed by an investigation of key OPO properties and their dependence on various physical parameters. In addition, we present the dynamics of these OPO's, identifying regimes of steady-state, multistability, period doubling, and chaotic behavior. 


\section{SECOND-ORDER NONLINEAR INTERACTIONS}

The phase-matched second-order nonlinear interaction between three collinear monochromatic plane waves

$$
E_{m}(z, t)=\operatorname{Re}\left[E_{m} \exp \left(j\left(\omega_{m} t-k_{m} z\right)\right)\right] \quad m=1,2,3
$$

with complex field amplitudes $E_{m}$ are described by the coupled-mode equations [21]

$$
\begin{aligned}
& \frac{d E_{1}}{d z}=-j \frac{\omega_{1} d_{e}}{n_{1} c} E_{3} E_{2}^{*} \\
& \frac{d E_{2}}{d z}=-j \frac{\omega_{2} d_{e}}{n_{2} c} E_{3} E_{1}^{*} \\
& \frac{d E_{3}}{d z}=-j \frac{\omega_{3} d_{e}}{n_{3} c} E_{1} E_{2}
\end{aligned}
$$

where $d_{e}$ is the effective nonlinear coefficient, $n_{m}$ are the refractive indices, and the frequencies are related by $\omega_{3}=$ $\omega_{1}+\omega_{2}$. The initial conditions at the input facet of the nonlinear crystal determine whether the interaction results in second-harmonic, sum-frequency, or parametric generation.

A pump field at $\omega_{3}$ and a signal field at $\omega_{2}$ at the input result in an OPA, where the signal is amplified and, in the process, an idler field at $\omega_{1}$ is generated [21]. The parametric gain experienced by the signal field saturates with increasing signal intensity. A singly resonant OPO is formed by placing the OPA inside an optical cavity that is resonant at the signal frequency. The signal field builds up from noise if the unsaturated gain is higher than all cavity losses combined. In the steady state, the intracavity signal intensity assumes such a value that the saturated gain compensates for the cavity losses exactly. The idler leaves the cavity through a dichroic beamsplitter that is highly transmitting at the idler frequency.

In an OPA, the lack of an idler field at the input of the crystal results in field solutions whose intensities are independent of the relative phases of the pump and the signal; the generated idler adjusts its phase to compensate for the phase fluctuations in the input pump and signal beams. Utilizing this property, it is possible to convert the three coupled-mode equations for the complex field amplitudes to three real equations [20]. It is also convenient to define normalized field amplitudes $a_{m}$ such that $a_{m}^{2}$ represents the photon flux densities at each frequency $\omega_{m}$. In doing this, we choose the phases of $E_{m}$ such that the required phase relation for the OPA is satisfied, and we define the real and normalized amplitudes $a_{m}$ through

$$
\begin{aligned}
& E_{1}=-j \sqrt{2 \hbar \omega_{1} / n_{1} c \epsilon_{0}} a_{1} \\
& E_{2}=\sqrt{2 \hbar \omega_{2} / n_{2} c \epsilon_{0}} a_{2} \\
& E_{3}=\sqrt{2 \hbar \omega_{3} / n_{3} c \epsilon_{0}} a_{3} .
\end{aligned}
$$

The coupled-mode equations for the normalized field amplitudes can then be written in the form

$$
\begin{aligned}
& \frac{d a_{1}}{d z}=\kappa_{a} a_{3} a_{2} \\
& \frac{d a_{2}}{d z}=\kappa_{a} a_{3} a_{1} \\
& \frac{d a_{3}}{d z}=-\kappa_{a} a_{1} a_{2}
\end{aligned}
$$

where the coupling constant is defined as

$$
\kappa_{a}=d_{e} \sqrt{\frac{2 \hbar}{c^{3} \epsilon_{0}}} \sqrt{\frac{\omega_{1} \omega_{2} \omega_{3}}{n_{1} n_{2} n_{3}}} .
$$

For convenience, we further assume that the pump and signal field amplitudes at the crystal entrance are both positive. The solutions of (8)-(10) are then given in terms of Jacobi elliptic functions as [8], [21]

$$
\begin{aligned}
& a_{1}(z)=\sqrt{C_{1}} \operatorname{cn}\left(Z_{a} \mid m_{a}\right) \\
& a_{2}(z)=\sqrt{C_{2}} \operatorname{dn}\left(Z_{a} \mid m_{a}\right) \\
& a_{3}(z)=\sqrt{C_{1}} \operatorname{sn}\left(Z_{a} \mid m_{a}\right)
\end{aligned}
$$

where

$$
\begin{aligned}
& C_{1}=a_{1}^{2}(z)+a_{3}^{2}(z)=a_{3}^{2}(0) \\
& C_{2}=a_{2}^{2}(z)+a_{3}^{2}(z)=a_{2}^{2}(0)+a_{3}^{2}(0)
\end{aligned}
$$

are the Manley-Rowe [22] conserved quantities,

$$
\begin{aligned}
m_{a} & =\frac{C_{1}}{C_{2}} \\
Z_{a} & =K\left(m_{a}\right)-\kappa_{a} \sqrt{C_{2}} z
\end{aligned}
$$

and

$$
K(m)=\int_{0}^{\pi / 2}\left(1-m \sin ^{2} \theta\right)^{-1 / 2} d \theta
$$

is the definition of the quarter period of Jacobi elliptic functions [23].

In SFG, a lower frequency input at $\omega_{4}$ and a higher frequency input at $\omega_{5}$ result in a sum-frequency field at $\omega_{6}=$ $\omega_{4}+\omega_{5}$. As in the OPA case, the lack of a sum-frequency field at the input allows us to write the coupled-mode equations in terms of real field amplitudes using

$$
\begin{aligned}
& E_{4}=\sqrt{2 \hbar \omega_{4} / n_{4} c \epsilon_{0}} a_{4} \\
& E_{5}=\sqrt{2 \hbar \omega_{5} / n_{5} c \epsilon_{0}} a_{5} \\
& E_{6}=-j \sqrt{2 \hbar \omega_{6} / n_{6} c \epsilon_{0}} a_{6} .
\end{aligned}
$$

In this case, the coupled-mode equations become

$$
\begin{aligned}
& \frac{d a_{4}}{d z}=-\kappa_{b} a_{6} a_{5} \\
& \frac{d a_{5}}{d z}=-\kappa_{b} a_{6} a_{4} \\
& \frac{d a_{6}}{d z}=\kappa_{b} a_{4} a_{5}
\end{aligned}
$$

with the coupling constant

$$
\kappa_{b}=d_{e} \sqrt{\frac{2 \hbar}{c^{3} \epsilon_{0}}} \sqrt{\frac{\omega_{4} \omega_{5} \omega_{6}}{n_{4} n_{5} n_{6}}} .
$$

The solutions for the SFG process depend on which of the two input fields has greater photon flux density at the crystal entrance. If $a_{4}^{2}(0)>a_{5}^{2}(0)$, the solutions become [8]

$$
\begin{aligned}
& a_{4}(z)=\sqrt{C_{4}} \operatorname{dn}\left(Z_{b} \mid m_{b}\right) \\
& a_{5}(z)=\sqrt{C_{5}} \operatorname{cn}\left(Z_{b} \mid m_{b}\right) \\
& a_{6}(z)=\sqrt{C_{5}} \operatorname{sn}\left(Z_{b} \mid m_{b}\right)
\end{aligned}
$$


TABLE I

Phase-Matching Geometries for the SF-OPO

\begin{tabular}{|c|c|c|c|c|}
\hline & \multicolumn{2}{|c|}{ OPO } & \multicolumn{2}{|c|}{ SFG } \\
\hline Type & $\omega_{3}$ & $\omega_{1}+\omega_{2}$ & $\omega_{2}+\omega$ & $\rightarrow \omega_{6}$ \\
\hline I & $f-$ & $s+s$ & $s+s$ & $\rightarrow f$ \\
\hline II & $f$ & $f+s$ & $f+s$ & $\rightarrow f$ \\
\hline III & $f-$ & $s+f$ & $s+f$ & $\rightarrow f$ \\
\hline Case & OPO & SFG & Rotation & Class \\
\hline 1 & I & I & pump & C \\
\hline 2 & II & I & pump & $\mathrm{C}$ \\
\hline 3 & III & I & both & B \\
\hline 4 & I & II & both & B \\
\hline 5 & II & II & both & B \\
\hline 6 & III & II & pump & C \\
\hline 7 & I & III & none & A \\
\hline 8 & II & III & none & A \\
\hline 9 & III & III & signal & D \\
\hline
\end{tabular}

where

$$
\begin{aligned}
& C_{4}=a_{4}^{2}(z)+a_{6}^{2}(z)=a_{4}^{2}(0) \\
& C_{5}=a_{5}^{2}(z)+a_{6}^{2}(z)=a_{5}^{2}(0)
\end{aligned}
$$

are the Manley-Rowe conserved quantities and

$$
\begin{aligned}
Z_{b} & =\kappa_{b} \sqrt{C_{4}} z \\
m_{b} & =\frac{C_{5}}{C_{4}} .
\end{aligned}
$$

The solutions for the case where $a_{5}^{2}(0)>a_{4}^{2}(0)$ are obtained by interchanging the field subscripts 4 and 5 and the Manley-Rowe subscripts 1 and 2.

\section{Sum-Frequency Generating OPO (SF-OPO)}

The single-crystal sum-frequency generating OPO (SFOPO) is based on the premise that optical parametric generation and SFG can both be phase matched for the same direction of propagation inside the nonlinear crystal. This simultaneous phase-matching condition may be achieved with either BPM or QPM. Furthermore, a number of different polarization geometries are possible, depending on the types of OPO and SFG phase matching. Some of these geometries require a polarization rotation for the signal or the pump fields.

\section{A. Birefringent Phase Matching (BPM)}

Table I and Fig. 1 together summarize all polarization geometries that can potentially be phase matched with BPM for a single-crystal SF-OPO. We follow the convention that the fields are labeled according to $\omega_{1}<\omega_{2}<\omega_{3}$ [24]. The field at $\omega_{3}$ is the OPO pump. The assignment of the "signal" label to $\omega_{1}$ or $\omega_{2}$ is somewhat arbitrary. In this paper, the resonant field in the OPO cavity, which also constitutes the lower frequency
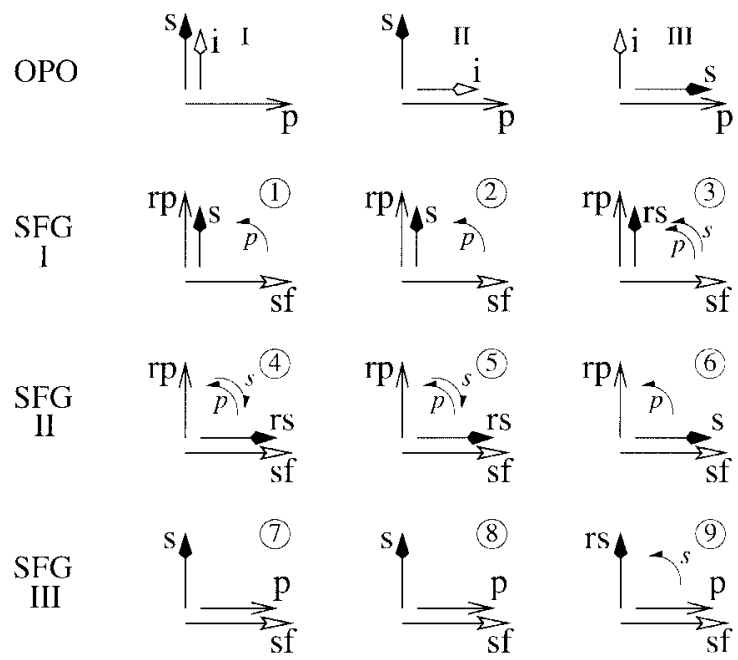

Fig. 1. Polarization diagrams for all possible SF-OPO geometries. The fast axis is horizontal and the slow axis is vertical. Polarizations for the pump (p), signal (s), idler (i), polarization rotated signal (rs), polarization rotated pump (rp), and sum-frequency (sf) fields are shown. Polarization rotation is indicated with an arc where required. Each combination of phase-matching types for the OPO and SFG processes is labeled with a circled number.

input field for the SFG process, is called the "signal" and is labeled as being at $\omega_{2}$. However, our formulation and results are equally valid if the field at $\omega_{1}$ is resonated and used as an SFG input.

For BPM in materials exhibiting normal dispersion, the highest frequency fields of both interactions $\left(\omega_{3}\right.$ and $\omega_{6}=$ $\omega_{2}+\omega_{3}$ ) have to be polarized along the fast axis of the crystal. In a type-I OPO, both the signal and the idler are polarized along the slow axis, whereas in a type-II (III) OPO, the signal is along the slow (fast) axis and the idler is along the fast (slow) axis. Type-I SFG has both input fields along the slow axis, whereas type-II (III) SFG has the lower frequency input along the fast (slow) axis and the higher frequency input along the slow (fast) axis.

There are nine possible cases corresponding to different combinations of birefringent phase-matching types for the OPO and SFG, as shown in Table I and Fig. 1. In cases 7 and 8, the pump and the signal fields are polarized along the same axes in both the OPO and SFG processes. As a result, the two processes become coupled through the signal and pump fields. The set of coupled-mode equations that describe this interaction are

$$
\begin{aligned}
& \frac{d a_{1}}{d z}=\kappa_{a} a_{3} a_{2} \\
& \frac{d a_{2}}{d z}=\kappa_{a} a_{3} a_{1}-\kappa_{b} a_{6} a_{3} \\
& \frac{d a_{3}}{d z}=-\kappa_{a} a_{1} a_{2}-\kappa_{b} a_{6} a_{2} \\
& \frac{d a_{6}}{d z}=\kappa_{b} a_{2} a_{3} .
\end{aligned}
$$

We arrive at these equations by combining the OPO equations [(8)-(10)] with the SFG equations [(23)-(25)]. The signal $a_{2}$ (pump $a_{3}$ ) and the lower frequency SFG input $a_{4}$ (higher frequency SFG input $a_{5}$ ) are the same field mode; the rate of change of the signal mode is the sum of the rates of change 
of the OPO signal and the lower frequency SFG input fields separately. Similarly, the rate of change of the pump mode is the sum of the rates of change of the OPO pump and the higher frequency SFG input fields. The same result can be obtained by considering the total nonlinear polarization fields $P_{2}$ and $P_{3}$ at $\omega_{2}$ and $\omega_{3}$, respectively, and rederiving the coupledmode equations. We designate this SF-OPO as class A, using a classification similar to self-doubling OPO's [20].

In cases 3-5, the pump and signal fields are both orthogonally polarized between the OPO and SFG processes. The same crystal can be used for both processes at the same time through extracavity polarization rotation of the pump and intracavity polarization rotation of the signal. However, the two processes are not coupled in the crystal as they are in class-A interactions, and the coupled-mode equations that govern this SF-OPO are simply (8)-(10) and (23)-(25). We designate this SF-OPO as class B.

In cases 1,2 , and 6 , the pump field is polarized orthogonally between the OPO and SFG processes. A polarization rotation of the pump at the cavity input is required for SFG to take place. The signal field is common to both processes and couples them to each other. The set of coupled-mode equations that describes all three cases is

$$
\begin{aligned}
& \frac{d a_{1}}{d z}=\kappa_{a} a_{3} a_{2} \\
& \frac{d a_{2}}{d z}=\kappa_{a} a_{3} a_{1}-\kappa_{b} a_{6} a_{5} \\
& \frac{d a_{3}}{d z}=-\kappa_{a} a_{1} a_{2} \\
& \frac{d a_{5}}{d z}=-\kappa_{b} a_{6} a_{2} \\
& \frac{d a_{6}}{d z}=\kappa_{b} a_{2} a_{5} .
\end{aligned}
$$

We designate this SF-OPO process as class $\mathrm{C}$.

In case 9 , the signal is orthogonally polarized between the OPO and SFG processes, and an intracavity polarization rotation of the signal is necessary. The OPO and SFG processes are coupled to each other through the pump, which is common to both processes inside the crystal. The coupled-mode equations that describe the interaction are

$$
\begin{aligned}
& \frac{d a_{1}}{d z}=\kappa_{a} a_{3} a_{2} \\
& \frac{d a_{2}}{d z}=\kappa_{a} a_{3} a_{1} \\
& \frac{d a_{3}}{d z}=-\kappa_{a} a_{1} a_{2}-\kappa_{b} a_{6} a_{4} \\
& \frac{d a_{4}}{d z}=-\kappa_{b} a_{6} a_{3} \\
& \frac{d a_{6}}{d z}=\kappa_{b} a_{3} a_{4} .
\end{aligned}
$$

We designate this SF-OPO process as class D.

For each combination of phase-matching types for the OPO and SFG processes, the respective coupling constants $\kappa_{a}$ and $\kappa_{b}$ depend on the phase-matched frequencies, the refractive indices, and the effective nonlinear coefficients. The ratio of the two coupling constants $\beta=\kappa_{b} / \kappa_{a}$ is an important quantity that may assume a range of values depending on these parameters. Here, the relative magnitudes of the frequencies and effective nonlinear coefficients are of particular importance. If the OPO and SFG processes are of the same phasematching type, the effective nonlinear coefficients differ only due to dispersion [24]. However, for different phase-matching types, the effective nonlinear coefficients may be dramatically different from each other, since they have different functional dependences on the elements of the second-order nonlinear tensor.

\section{B. Quasi-Phase Matching (QPM)}

QPM offers the potential to phase match a wide range of wavelengths for any second-order nonlinear interaction by employing periodic domain reversals in ferroelectric crystals. These domain reversals lead to a periodic modulation of the effective nonlinear coefficient, and a particular spatial harmonic of the modulation compensates for the phase mismatch [25]. In single-process frequency conversion applications, the first-order spatial harmonic is usually chosen, as this provides the largest effective nonlinear coefficient. It is also customary to have all fields to be polarized in the same direction to take advantage of a large diagonal element of the nonlinear susceptibility tensor. However, it is possible to use QPM with orthogonally polarized fields as well. There are eight potential phase-matching types for QPM, as opposed to the three in BPM, since the highest frequency field of the interaction no longer has to be polarized along the fast axis of the crystal.

In terms of simultaneous phase matching of SFG in an OPO, the eight QPM types lead to 64 different combinations. We found that each of these combinations can be identified with one of the four classes introduced above, depending on whether the coupling between the two processes in the crystal is through the signal (class C), the pump (class D), both (class A), or neither (class B).

The QPM order of the two processes may or may not be different from each other. Since the effective nonlinear coefficient for a process depends on the QPM order as well as the other parameters mentioned above, choosing different QPM orders for the two processes provides a mechanism for adjusting the value of $\beta$. The results of Section V show that having some control on the value of $\beta$ can be very useful in maximizing the conversion efficiency of some SF-OPO's.

Note that QPM can easily be used to phase match two interactions in a single crystal by employing two consecutive sections with different poling periods. Such a double-grating PPLN was recently used for intracavity SFG of an OPO [26]. However, the dynamics of these double-grating devices are identical to those with two different crystals [7], [8].

\section{Single-PASS SOLUTIONS}

In order to analyze the performance of SF-OPO's, it is first necessary to calculate the single-pass saturated parametric gain experienced by the signal field by solving the coupled-mode equations for each class. A simple transformation maps the class-A equations to regular OPA equations, leading to analytical solutions in terms of Jacobi elliptic functions. In class-B 
SF-OPO's, the OPA and SFG processes are not coupled, and analytical solutions are readily available. For class-C and classD SF-OPO's, however, we resorted to numerical techniques to solve the coupled-mode equations. These solutions were computed using the Runge-Kutta-Fehlberg method [27].

\section{A. Class-A Solutions}

An important simplification in the coupled-mode equations for class-A SF-OPO's is achieved by recognizing that the right-hand sides of (34) and (37) are proportional to each other. Since both the idler field $a_{1}$ and the sum-frequency field $a_{6}$ have zero amplitude at the crystal input, we have $a_{6}=\beta a_{1}$ for all values of $z$. The coupled-mode equations then become

$$
\begin{aligned}
& \frac{d a_{1}}{d z}=\kappa_{a} a_{3} a_{2} \\
& \frac{d a_{2}}{d z}=\left(1-\beta^{2}\right) \kappa_{a} a_{3} a_{1} \\
& \frac{d a_{3}}{d z}=-\left(1+\beta^{2}\right) \kappa_{a} a_{1} a_{2} .
\end{aligned}
$$

The evolution of the field amplitudes as they propagate down the crystal depends on the value of $\beta$. If $\beta$ is equal to unity, the right-hand side of (49) becomes equal to zero for all values of $z$. In this case, the parametric gain provided by the pump is exactly balanced by the nonlinear loss due to SFG, and the signal field amplitude stays constant throughout the length of the crystal. If $\beta$ is less than unity, the parametric gain overcomes the nonlinear SFG loss, and the signal field is amplified. Under this condition, the substitutions $a_{1}=u_{1} / \sqrt{\left(1-\beta^{2}\right)\left(1+\beta^{2}\right)}, a_{2}=u_{2} / \sqrt{1+\beta^{2}}$, and $a_{3}=u_{3} / \sqrt{1-\beta^{2}}$ transform (48)-(50) to the coupledmode equations for a regular OPA [(8)-(10)] in terms of the variables $u_{1}, u_{2}$, and $u_{3}$. After transforming the OPA solutions [(12)-(14)] in terms of $u_{1}, u_{2}$, and $u_{3}$ back to the original class-A variables $a_{1}, a_{2}$, and $a_{3}$, the evolution of the field amplitudes can be expressed as

$$
\begin{aligned}
& a_{1}(z)=\sqrt{\frac{C_{1}}{\left(1-\beta^{2}\right)\left(1+\beta^{2}\right)}} \operatorname{cn}\left(Z_{a} \mid m_{a}\right) \\
& a_{2}(z)=\sqrt{\frac{C_{2}}{1+\beta^{2}}} \operatorname{dn}\left(Z_{a} \mid m_{a}\right) \\
& a_{3}(z)=\sqrt{\frac{C_{1}}{1-\beta^{2}} \operatorname{sn}\left(Z_{a} \mid m_{a}\right)} \\
& a_{6}(z)=\beta a_{1}(z)
\end{aligned}
$$

where

$$
\begin{aligned}
& C_{1}=\left(1-\beta^{2}\right)\left(1+\beta^{2}\right) a_{1}^{2}(z)+\left(1-\beta^{2}\right) a_{3}^{2}(z) \\
& C_{2}=\left(1+\beta^{2}\right) a_{2}^{2}(z)+\left(1-\beta^{2}\right) a_{3}^{2}(z)
\end{aligned}
$$

are the class-A Manley-Rowe conserved quantities [(15) and (16)] expressed in terms of the field amplitudes $a_{1}, a_{2}$, and $a_{3}$. Here, $m_{a}$ and $Z_{a}$ are as defined in (17) and (18), respectively.

If $\beta$ is larger than unity, the parametric gain provided by the pump cannot compensate for the nonlinear SFG loss. In this case, the net gain experienced by the signal field is always less than or equal to unity, regardless of the values of $\kappa_{a}$ and the input photon flux densities. Therefore, it is impossible for the SF-OPO to get above threshold.

\section{B. Class-B Solutions}

In class-B SF-OPO's, the OPO signal (pump) and the SFG lower (higher) frequency input fields have orthogonal polarizations. Therefore, the OPA and SFG processes are completely independent of each other in a single pass through the crystal. However, an intracavity polarization rotation of the signal field and an extracavity polarization rotation of the pump field with the use of half-wave retarders can couple the two processes and allow SFG to take place. The single-pass solutions are given in (12)-(14) and (27)-(29).

\section{Class-C Solutions}

Since analytical solutions of class-C coupled-mode equations [(38)-(42)] are not available, we resorted to numerical techniques to compute the evolution of the field amplitudes and the net signal gain. In these calculations, we used the Runge-Kutta-Fehlberg method, which is an adaptive stepsize method commonly used for solving ordinary differential equations [27].

In our calculations, we found that the field amplitudes oscillate either periodically or aperiodically as a function of $z$, depending on the initial values and $\beta$. This behavior can best be analyzed by transforming the coupled-mode equations [(38)-(42)] to a single differential equation [20]. In doing this, we use the class-C Manley-Rowe conserved quantities

$$
\begin{aligned}
& C_{1}=a_{1}^{2}(z)+a_{3}^{2}(z)=a_{3}^{2}(0) \\
& C_{2}=a_{2}^{2}(z)+a_{3}^{2}(z)+a_{6}^{2}(z)=a_{2}^{2}(0)+a_{3}^{2}(0) \\
& C_{3}=a_{5}^{2}(z)+a_{6}^{2}(z)=a_{5}^{2}(0)
\end{aligned}
$$

and define new variables $\theta(z)$ and $\gamma(z)$ through $a_{1}=$ $\sqrt{C_{1}} \sin \theta, a_{3}=\sqrt{C_{1}} \cos \theta, a_{5}=\sqrt{C_{3}} \cos \gamma$, and $a_{6}=$ $\sqrt{C_{3}} \sin \gamma$. Since no idler or sum-frequency is present at the crystal input, $\theta(0)$ and $\gamma(0)$ are equal to an integral multiple of $2 \pi$; for convenience, we choose $\theta(0)=\gamma(0)=0$. Substituting into (38) and (42), we obtain

$$
a_{2}(z)=\frac{1}{\kappa_{a}} \frac{d \theta}{d z}
$$

and $\gamma(z)=\beta \theta(z)$. These equations, along with the Manley-Rowe relation for $C_{2}$, allow us to reduce the set of coupled-mode equations to a single differential equation

$$
\frac{1}{\kappa_{a}^{2}}\left(\frac{d \theta}{d z}\right)^{2}+C_{1} \cos ^{2} \theta+C_{3} \sin ^{2}(\beta \theta)=C_{2}
$$

in the variable $\theta(z)$. The solution of this equation either oscillates periodically around zero or increases monotonically, depending on the values of the Manley-Rowe quantities and $\beta$. If $C_{1} \cos ^{2} \theta+C_{3} \sin ^{2}(\beta \theta)$ is larger than $C_{2}$ for any value of $\theta$, the field amplitudes oscillate periodically. This condition is roughly equivalent to $a_{2}^{2}(0)<a_{5}^{2}(0)$ (depending on whether $\beta$ is rational or not). In this case, the signal field is fully depleted inside the crystal, with the depletion locations coinciding with the maxima and minima of $\theta(z)$. In the other case where 
$\theta(z)$ increases monotonically, the field amplitudes are periodic functions of $z$ only if $\beta$ has a rational value; otherwise, the field amplitudes vary aperiodically [20].

Although (61) gives more insight into the qualitative behavior of the solutions, in calculating the single-pass signal gain we prefer to solve (38)-(42) directly. The first term of (61) brings about an ambiguity in the sign of $d \theta / d z$, and every time the signal field is depleted the corresponding sign change in $d \theta / d z$ must be taken into account.

\section{Class-D Solutions}

Calculation of the class-D field amplitudes and signal gain necessitates the use of numerical techniques as well. We observed that the field amplitudes oscillate periodically in $z$, regardless of the values of the Manley-Rowe quantities and $\beta$. This behavior is easier to analyze if the coupled-mode equations are reduced to a single differential equation similar to (61). Furthermore, this differential equation can be used to obtain conditions that maximize conversion efficiency, as outlined in Section V.

The Manley-Rowe conserved quantities for class-D SFOPO's are

$$
\begin{aligned}
& C_{1}=a_{2}^{2}(z)-a_{1}^{2}(z)=a_{2}^{2}(0) \\
& C_{2}=a_{2}^{2}(z)+a_{3}^{2}(z)+a_{6}^{2}(z)=a_{2}^{2}(0)+a_{3}^{2}(0) \\
& C_{3}=a_{4}^{2}(z)+a_{6}^{2}(z)=a_{4}^{2}(0) .
\end{aligned}
$$

We define new variables $\theta(z)$ and $\gamma(z)$ through $a_{1}=$ $\sqrt{C_{1}} \sinh \theta, a_{2}=\sqrt{C_{1}} \cosh \theta, a_{4}=\sqrt{C_{3}} \cos \gamma$, and $a_{6}=\sqrt{C_{3}} \sin \gamma$. Since $a_{1}(0)=a_{6}(0)=0, \theta(0)=0$ and $\gamma(0)$ is an integral multiple of $2 \pi$; we choose $\gamma(0)=0$ for convenience. When these transformations are substituted into the coupled-mode equations (43) and (47), the pump field amplitude is obtained in terms of $\theta(z)$ or $\gamma(z)$ as

$$
a_{3}(z)=\frac{1}{\kappa_{a}} \frac{d \theta}{d z}=\frac{1}{\kappa_{b}} \frac{d \gamma}{d z}
$$

It is then possible to integrate this equation and get a fourth conserved quantity $C_{4}=\theta(z) / \kappa_{a}-\gamma(z) / \kappa_{b}=0$. Using this relation and the Manley-Rowe relation for $C_{2}$, we obtain a single differential equation

$$
\frac{1}{\kappa_{a}^{2}}\left(\frac{d \theta}{d z}\right)^{2}+C_{1} \cosh ^{2} \theta+C_{3} \sin ^{2}(\beta \theta)=C_{2}
$$

in the variable $\theta(z)$. As $\theta(z)$ starts from zero at the crystal entrance and increases, the second term in (66) increases monotonically until $d \theta / d z$ becomes zero. It is seen from (65) that this point corresponds to complete pump depletion. Since the first term of (66) cannot be negative, $\theta(z)$ has to decrease from this point on. Hence, the solution of (66) oscillates periodically around zero, and the field amplitudes also evolve periodically with the period being identified by complete pump depletion. As in class-C single-pass solutions, we prefer to solve the original coupled-mode equations [(43)-(47)] in computing the single-pass signal gain.

\section{SF-OPO DYNAMICS}

A singly resonant SF-OPO is constructed by placing the OPA crystal inside a cavity that is resonant at the signal frequency. The pump field enters the cavity through a dichroic mirror that is highly transmissive at that wavelength. The cavity typically has some residual linear loss $(L)$ at the signal wavelength due to less than unity reflectivities of the cavity mirrors and imperfect antireflection coatings on the nonlinear crystal and other transmissive optics. This linear loss can also be represented by a lumped cavity reflectance $R=1-L$. Ordinarily, no output coupling through a partially reflecting mirror is necessary for the signal field; the conversion to the sum-frequency field provides a nonlinear output coupling mechanism.

The parametric gain of the OPA is related to a number of parameters, some of which can conveniently be lumped into a single dimensionless quantity, called the nonlinear drive, which is a measure of the strength of the parametric interaction [6], [8], [20]. The nonlinear drive is defined as $D=\left(\kappa_{a} a_{p} l\right)^{2}$, where $a_{p}^{2}$ is the total pump photon flux density at the crystal entrance and $l$ is the crystal length. In classes $A$ and D, where there is no pump polarization rotation, we have $a_{p}^{2}=a_{3}^{2}(0)$, whereas in classes B and C $a_{p}^{2}=a_{3}^{2}(0)+a_{5}^{2}(0)$ is equal to the total pump photon flux density before polarization rotation.

The nonlinear drive $D_{\text {th }}$ for which the small-signal (unsaturated) gain $g_{0}$ compensates the cavity losses exactly $\left(g_{0} R=1\right)$ defines the threshold point. For a particular nonlinear drive above threshold, there is sufficient gain to overcome the cavity losses, and the intracavity signal field begins to build up from noise (parametric fluorescence). The time evolution of the intracavity signal field depends on the value of the nonlinear drive and how the parametric gain saturates with increasing signal flux density. To investigate the dynamics of SF-OPO's, an iterative method that simulates the intracavity build-up of the signal photon flux can be used [8]. Since the OPA gain is a nonlinear mapping of the signal flux density from the input to the output with the cavity providing a feedback path with some loss, the SF-OPO can be viewed as a discrete dynamical system [28], [29].

In this section, we investigate the dynamics of SF-OPO's using a numerical iterative approach. The behavior of a particular SF-OPO depends on a number of physical parameters, all of which can be characterized by the nonlinear drive $D$, the ratio of the two coupling constants $\beta$, the residual cavity loss $L$, and the polarization rotation angle $\alpha$ (where applicable). We have restricted our analysis to a range of parameter values that are likely to be encountered in practical experiments. However, our aim here is not to model an experiment accurately, but to bring out the fundamental properties of SF-OPO's using a simple plane-wave approach. Our analysis does not take into account many experimental realities such as the Gaussian beam nature of the fields, the temporal profile of the pulses, group velocity mismatch between field components, group velocity dispersion, or beam walk-off.

The nonlinear drive $D=\left(\kappa_{a} a_{p} l\right)^{2}$ can take on a wide range of values depending on various physical parameters. The operation wavelengths together with the linear and nonlinear 
optical properties of the crystal determine $\kappa_{a}$ according to (11). The crystal (or the interaction) length $l$ may be limited by growth constraints or pulse propagation characteristics such as group velocity mismatch and/or dispersion [10]. The available power (average for $\mathrm{CW}$, peak for pulsed) from the pump laser and beam focusing constraints determine the pump intensity at the crystal. Considering a range of values that these physical parameters may take in currently feasible experiments, we have decided to consider a range of 0.1 to 100 for the nonlinear drive. The lower bound for the nonlinear drive is mostly dictated by the OPO threshold and the upper bound by the available effective nonlinear coefficient, crystal length, and pump laser power.

In principle,

$$
\beta=\frac{\kappa_{b}}{\kappa_{a}}=\frac{d_{e b}}{d_{e a}} \sqrt{\frac{\omega_{6}}{\omega_{1}}} \sqrt{\frac{n_{1} n_{2} n_{3}}{n_{4} n_{5} n_{6}}}
$$

may assume any nonnegative value. For $\beta \ll 1$, the SF-OPO becomes an ordinary OPO. When $\beta \simeq 1$, the strength of the two interactions are comparable to each other. Since the last factor containing the refractive indices in (67) is approximately equal to unity, the value of $\beta$ is determined by the ratio of the two effective nonlinear coefficients and the ratio of the idler and sum-frequency wavelengths. As pointed out in Section III, the two nonlinear coefficients differ only due to dispersion if the OPO and the SFG processes are of the same phasematching type. This situation leads to $d_{e b} / d_{e a} \approx 1$ in the cases of BPM and QPM with both processes having the same QPM order. If the phase-matching types and/or the QPM orders are different, $d_{e b} / d_{e a}$ may have any value. However, it is not likely for $d_{e b} / d_{e a}$ to be much greater than unity in any case, since this would imply a smaller nonlinear drive for the OPO process, making it difficult to get above threshold. On the other hand, the $\sqrt{\omega_{6} / \omega_{1}}$ term has to be greater than $\sqrt{3}$ (since $\omega_{6}=2 \omega_{2}+\omega_{1}>3 \omega_{1}$ ), and less than 3 , being limited by the transparency range of the nonlinear crystal. Taking all these factors into account, we conclude that $\beta$ would be in the $0.3-3$ range in most practical situations.

The residual cavity losses depend on the linear absorption coefficient of the nonlinear crystal and the quality of the mirrors, antireflection coatings, and other intracavity optics. In most cases, it is desirable to keep this loss as small as possible. In practice, $L$ is usually limited to a few percent at most. In our calculations, we have chosen to take $L=0.04$ as a conservative value. Our results have shown that the qualitative behavior of a typical SF-OPO does not change very much with variations in $L$.

The performance of the SF-OPO is best characterized by the photon conversion efficiency. Since two pump photons are needed to generate one sum-frequency photon, the conversion efficiency is the ratio of twice the output sum-frequency photon flux density to the total input pump photon flux density, $\eta=$ $2 a_{6}^{2}(l) / a_{p}^{2}$. The conversion efficiency represents the overall efficiency of the two-step process (from the pump to the signal and then to the sum-frequency) and is equal to unity for the case of total conversion in the steady state.

In our calculations, we start out with an initial signal photon flux density to represent parametric fluorescence [30]. We then iterate this through the system several times, in effect multiplying the input flux density with the saturated signal gain $g$ and cavity reflectance $R$ at each iteration. The turn-on transient is usually contained in the first 500 to $10^{4}$ iterations. After this transient, the SF-OPO may converge to a stable steady-state value, oscillate between two or more constant values, or exhibit chaotic behavior.

When the iterations converge to a stable steady-state intracavity signal flux density, this steady-state value $a_{2}^{2}(0)$ is a solution of

$$
g\left[a_{2}^{2}(0)\right]=1 / R
$$

since this is the signal flux density for which the linear cavity losses are exactly compensated by the OPA gain. However, (68) may have more than one solution, in which case we use graphical analysis [29] to determine which one of the solutions the iterations will converge to. Under certain circumstances, the steady-state solution becomes sensitive to the initial value, leading to a multistable behavior.

In our analysis of SF-OPO's, we consider the function

$$
f(x)=x R g(x)
$$

where $x=a_{2}^{2}(0)$. The solutions of (68) correspond to the fixed points of (69) which are the roots of $f(x)-x$. A fixed point $x_{n}$ is determined to be stable if the derivative of $f(x)$ at $x=x_{n}$ is less than unity, and unstable otherwise [29]. Fig. 2 shows plots of $f(x)$ that typify various situations that may be encountered. The $x_{0}=0$ point is always an unstable fixed point if the SF-OPO is above threshold. For the SFOPO of Fig. 2(a), the first nonzero fixed point $x_{1}$ is stable, there are no other fixed points, and the iterations converge to $x_{1}$. Increasing the nonlinear drive for the same SF-OPO in Fig. 2(b), we get two additional fixed points, the larger of the two new points $\left(x_{3}\right)$ being stable. (Note that there has to be at least one unstable fixed point in between any two stable fixed points.) However, it is impossible for the iterations to converge to $x_{3}$, since the peak value between the points $x_{0}$ and $x_{1}$ is less than $x_{2}$. In Fig. 2(c), where the nonlinear drive is increased further, however, the iterations may converge to either $x_{1}$ or $x_{3}$, depending on the initial value. For convergence to $x_{3}$, at least one of the iterates has to fall in the subset of points for which $f(x)>x_{2}$ in the $x_{0}<x<x_{1}$ interval. Going backward from this set to smaller values, we can determine which points near $x_{0}$ will converge to $x_{3}$. This analysis shows that, for points near $x_{0}$, a small perturbation in the initial value is enough to change the steady-state value, resulting in multistable behavior.

The presence of multistability may affect the operation of a practical SF-OPO in a number of ways. Each time the pump beam to a multistable SF-OPO is turned on, the intracavity signal power and hence the conversion efficiency converge to a different value, since the initial intracavity signal photon flux is slightly different. Furthermore, increasing and decreasing the pump intensity is expected to show a hysteresis behavior, with sudden jumps in the conversion efficiency occurring at different pump power levels, depending on whether the pump power is being increased or decreased. 


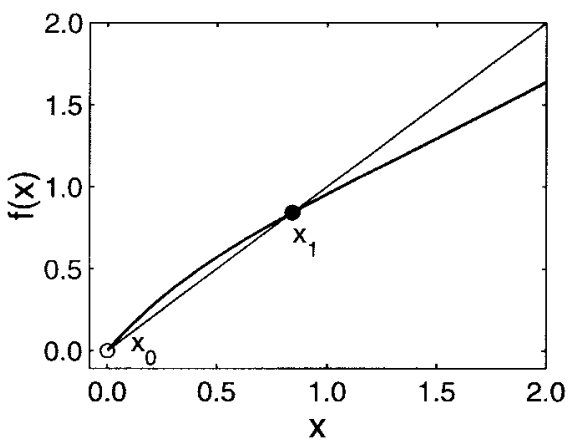

(a)

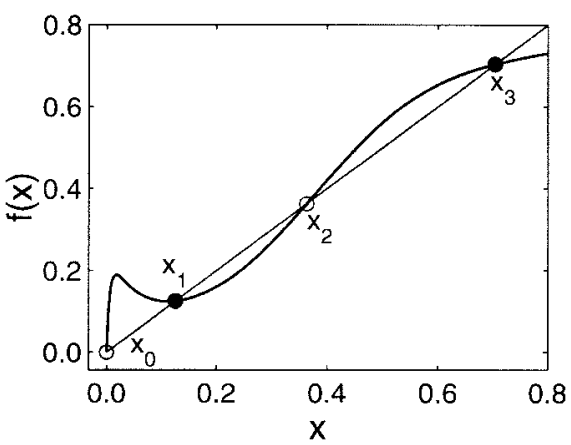

(b)

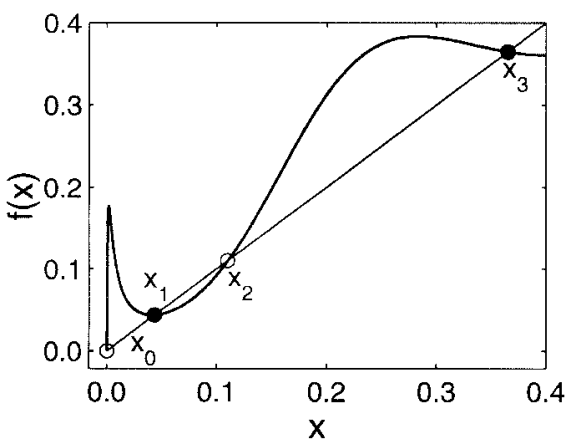

(c)

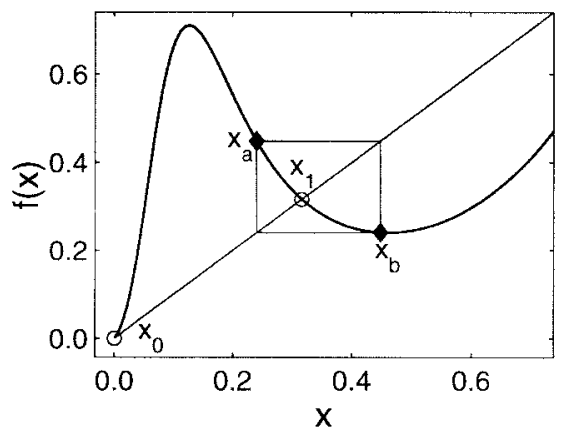

(d)

Fig. 2. Plots of $f(x)$ versus $x$ with both axes normalized to $a_{p}^{2}$. The stable (filled circles) and unstable (empty circles) fixed points are labeled. (a) Single stable fixed point $x_{1}$. (b) Two stable fixed points $x_{1}$ and $x_{3}$ with no multistability. (c) Two stable fixed points $x_{1}$ and $x_{3}$ with multistability. (d) Periodic oscillation with a period of two; $x_{a}$ and $x_{b}$ are stable fixed points of $f(f(x))$.

Periodic oscillations, where the iterations oscillate between two or more constant values, is another feature that may be observed in SF-OPO's. Fig. 2(d) shows an example of an oscillation with a period of two. In this situation, $f\left(x_{a}\right)=x_{b}$

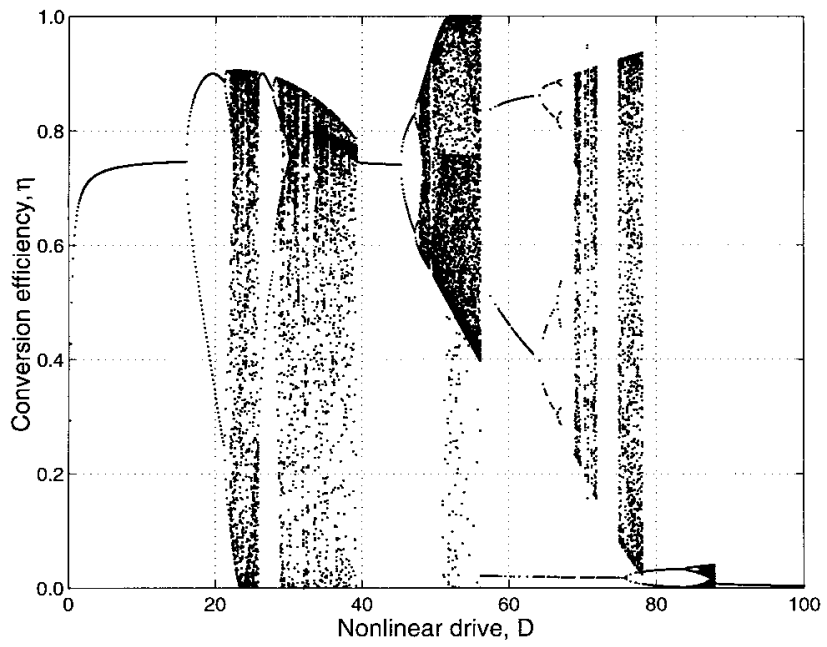

Fig. 3. Conversion efficiency as a function of the nonlinear drive for a class-C SF-OPO. ( $\beta=0.5, \alpha_{p}=45^{\circ}$, and $L=0.04$.)

and $f\left(x_{b}\right)=x_{a}$; in other words, $x_{a}$ and $x_{b}$ are fixed points of $f^{2}(x)=f(f(x))$ but not of $f(x)$. Typically, a stable fixed point, say $x_{1}$, becomes unstable as the nonlinear drive is increased, and a periodic oscillation with $x_{a}<x_{1}<x_{b}$ is formed, resulting in period doubling, as shown in Fig. 2(d). In general, periodic oscillations with any positive integer period is possible. In synchronously pumped pulsed OPO's, a periodic oscillation exhibits itself as a periodic output pulse sequence where each pulse in the sequence has a different magnitude. In CW OPO's, the cavity round-trip time and the bandwidth of parametric fluorescence determine the character of the periodic oscillations.

Period doubling is a common route to chaotic behavior in nonlinear systems [29]. SF-OPO's sometimes show chaotic oscillations, depending on the nonlinear drive, $\beta$, and polarization rotation angle. To identify periodic or chaotic oscillations at a particular set of values, we discard the transient and plot the next 64 consecutive iterates on a vertical line [8]. If the oscillation is periodic with period $m$, the 64 iterates accumulate at $m$ points. If the oscillations are chaotic, the iterates are distributed randomly. An example of such a plot is shown in Fig. 3. As the nonlinear drive is increased above threshold, the SF-OPO is at first stable and the conversion efficiency increases monotonically. Period doubling occurs around $D=16$, with consecutive doublings leading to chaotic oscillations. It is interesting to note that, when the SFOPO exhibits periodic or chaotic oscillations, the conversion efficiency may be greater than unity for some passes through the crystal and less than unity for others. However, the average of the conversion efficiency over all passes cannot be higher than unity in any case.

In synchronously pumped pulsed OPO's, a chaotic oscillation results in each output pulse having a different magnitude within the bounds determined by the iterations. In CW OPO's, the character of the chaotic oscillations are determined by the cavity round-trip time and the bandwidth of parametric fluorescence. On the other hand, it is not very likely for CW OPO's to reach the high nonlinear drive levels where chaotic oscillations come up. 


\section{A. Class-A Dynamics}

As pointed out in Section IV, class-A SF-OPO's can get above threshold only if $\beta$ is less than unity. Under this condition, the small-signal gain is

$$
g_{0}=\cosh ^{2} \sqrt{\left(1-\beta^{2}\right) D} .
$$

The SF-OPO threshold is found by solving $g_{0} R=1$ for $D$. The presence of the $\left(1-\beta^{2}\right)$ term indicates that the threshold nonlinear drive is influenced by the presence of the SFG process; this is in contrast with self-doubling OPO's [20].

The Manley-Rowe relation of (55) together with (54) shows that the conversion efficiency is maximized when the pump field is fully depleted at the end of the crystal. Taking the ratio of the Manley-Rowe relations [(55) and (56)] evaluated at $z=0$ and $z=l$, we find that $m_{a}=L$. The pump field has a $\operatorname{sn}(Z \mid m)$ functional dependence which assumes its zeros at even multiples of the Jacobi quarter period $K$. Therefore, complete pump depletion at $z=l$ requires that

$$
K-\kappa_{a} \sqrt{C_{2}} l=-2 n K, \quad n=0,1,2, \cdots .
$$

This relation leads to a family of optimum nonlinear drive values

$$
D_{\mathrm{opt}}=\frac{L}{1-\beta^{2}}[(2 n+1) K]^{2}
$$

that maximize the conversion efficiency for given values of $\beta$ and $L$. Only the smallest optimum nonlinear drive (corresponding to $n=0$ ) is of consequence, since for $n>0$ the iterations cannot converge to the stable fixed point that yields the maximum conversion efficiency by starting out from an initial value near zero. The ratio $D_{\text {opt }} / D_{\text {th }}$ depends only on $L$, similar to a regular OPO [6].

The nonlinear drive $D, \beta$, and $L$ uniquely determine the conversion efficiency of the SF-OPO. The conversion efficiency achieved at the optimum nonlinear drive

$$
\eta_{\max }=\frac{2 \beta^{2}}{1+\beta^{2}}
$$

is a function of $\beta$ only. As $\beta$ increases toward unity, the maximum conversion efficiency approaches unity, while the threshold and optimum nonlinear drive values increase without bound.

Fig. 4 shows the photon conversion efficiency as a function of the nonlinear drive for various $\beta$ values at a constant $L=0.04$. The peak of each curve is at $D_{\text {opt }}$ with a value of $\eta_{\max }$. Multistability is observed at large nonlinear drives in all cases, but shown for the $\beta=0.8$ case only. The minimum nonlinear drive value for which multistability is observed increases monotonically with both $\beta$ and $L$.

The cavity loss $L$ at the signal frequency results in the loss of signal photons from the cavity. One would expect that this useless loss should be minimized for maximum conversion to the sum-frequency. However, as illustrated in Fig. 5, the cavity losses can be adjusted to maximize the conversion efficiency, much like in a regular OPO. At a constant $\beta$ value of 0.8 , the conversion efficiency curve shifts to the right with increasing cavity loss, whereas the peak conversion efficiency does not change, in agreement with the analytical result given in (73).

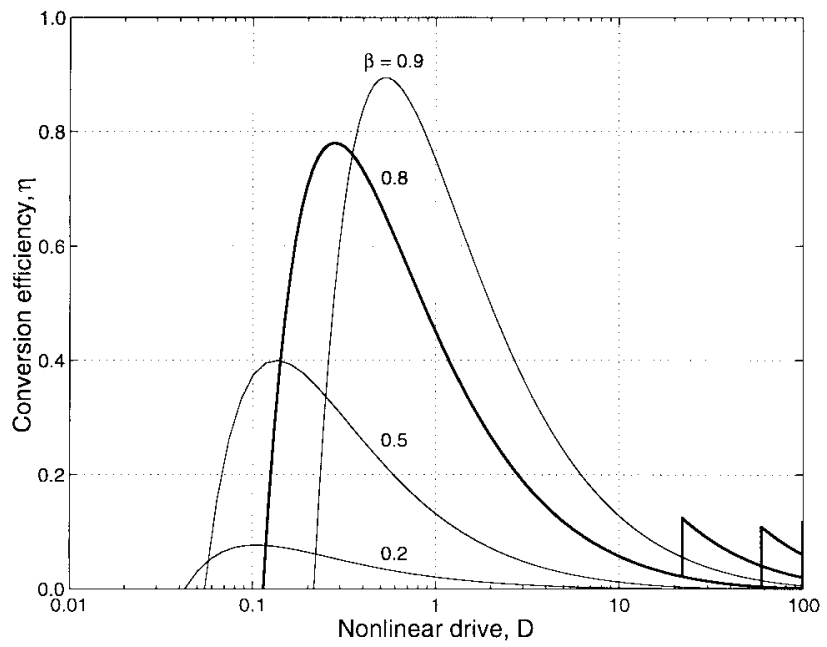

Fig. 4. Conversion efficiency as a function of the nonlinear drive for various values of $\beta$ for a class-A SF-OPO. For each case, $L=0.04$. Multistability exists in all cases but is shown only for $\beta=0.8$ (thick line).

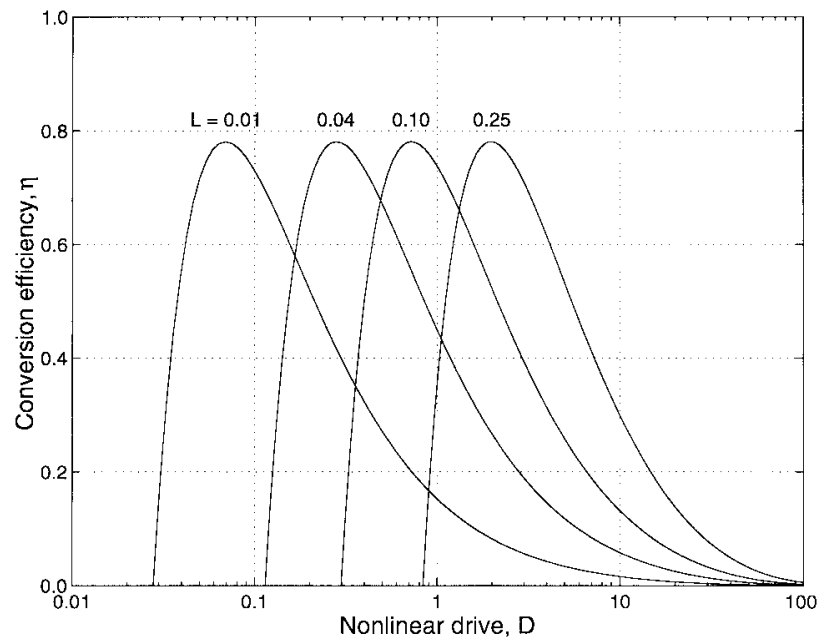

Fig. 5. Conversion efficiency as a function of the nonlinear drive for various values of $L$ for a class-A SF-OPO. For each case, $\beta=0.8$. Multistability exists but is not shown.

In the design of a class-A SF-OPO, the most important parameter is $\beta$. For high conversion efficiency, $\beta$ should be as high as possible without exceeding unity. However, if $\beta$ is very close to unity, then there is a risk that the SF-OPO will fall below threshold. In practice, a $\beta$ value in the range of $0.6-0.98$ should be sought to achieve $\eta>0.5$. Once $\beta$ is known, the target conversion efficiency is determined from (73), and the optimum cavity loss $L_{\mathrm{opt}}$ that maximizes the conversion efficiency is calculated using (72). If $L_{\mathrm{opt}}$ is less than the residual losses, the target conversion efficiency cannot be reached. However, in most cases, $L_{\mathrm{opt}}$ is likely to be greater than the residual losses $L_{r}$, and the SF-OPO can be optimized at the operating nonlinear drive with the use of an output coupler $\left(L_{\text {oC }}\right)$ for the signal field. (For example, in Fig. 5, at a modest nonlinear drive value of unity, a relatively large cavity loss of $L_{\mathrm{opt}}=0.14$ is needed for maximum conversion.)

The presence of an output coupler for the signal field results in a usable signal output from the SF-OPO. The conversion efficiency to the signal wavelength at the optimum value of 
cavity loss is

$$
\eta_{s}=\frac{L_{\mathrm{oc}} a_{2}^{2}(l)}{a_{p}^{2}(0)}=\frac{L_{\mathrm{oc}}}{L_{\mathrm{opt}}} \frac{1-\beta^{2}}{1+\beta^{2}}
$$

where $\left(1-L_{\mathrm{opt}}\right)=\left(1-L_{\mathrm{r}}\right)\left(1-L_{\mathrm{oc}}\right)$.

\section{B. Class-B Dynamics}

Class-B SF-OPO's require an intracavity polarization rotation for the signal and an extracavity polarization rotation for the pump fields. Two different modes of operation are possible depending on the intracavity polarization rotation scheme employed for the signal field. In the first case, the signal is rotated by an arbitrary amount $0^{\circ}<\alpha_{s}<90^{\circ}$, so that a fraction of $\sin ^{2} \alpha_{s}$ of the OPO signal flux is coupled to the lower frequency SFG field mode $a_{4}(0)$, while still maintaining a fraction of $\cos ^{2} \alpha_{s}$ as the input signal $a_{2}(0)$. At each pass through the crystal, the OPO signal $a_{2}$ experiences parametric gain, whereas the rotated signal $a_{4}$ is depleted due to SFG. Here, we assume that the residual rotated signal at the output of the crystal $a_{4}(l)$ is either coupled out of the cavity with the use of a polarizing beamsplitter or is negligible due to strong conversion. If, instead, both the signal and rotated signal fields are fed back to the input by the OPO cavity, the polarization mixing due to the intracavity retarder will result in the interference of the two fields in an uncontrolled fashion.

In this configuration, the OPO signal field mode experiences a linear loss of $\sin ^{2} \alpha_{s}$ in addition to other residual cavity losses. The total cavity reflectance for the OPO signal becomes $R \cos ^{2} \alpha_{s}$. There is no nonlinear output coupling mechanism in this situation, and the presence of the SFG process has no effect on the intracavity signal flux density $a_{2}^{2}$. Even though the SFG process is internal to the OPO cavity, the lower frequency SFG input at the signal wavelength is not resonant in the cavity. In effect, this configuration is equivalent to splitting the pump into two beams, downconverting one beam to the signal wavelength with a regular OPO of output coupler reflectivity $\sin ^{2} \alpha_{s}$, and then summing the other beam with the output of this OPO. Since the SFG process does not benefit from the high intracavity signal flux density, this configuration is not expected to be particularly efficient or useful.

On the other hand, a different mode of operation is achieved if the residual rotated signal is not coupled out of the cavity and $\alpha_{s}$ is set to $90^{\circ}$. In this scheme, the retarder switches around the polarizations of the output OPO signal $a_{2}(l)$ and the residual rotated signal $a_{4}(l)$ with no polarization mixing. As in the previous scheme, the polarization of the pump field is rotated before entering the cavity to provide the higher frequency SFG input field $a_{5}(0)$.

For a given extracavity pump polarization rotation angle of $\alpha_{p}$, maximum conversion to the sum-frequency requires complete depletion of the rotated pump field. From an experimental point of view, however, it is more meaningful to try to maximize the conversion efficiency at a constant nonlinear drive by adjusting $\alpha_{p}$. As an example, Fig. 6 shows the conversion efficiency and the depletion of the pump and the rotated pump flux densities as functions of $\alpha_{p}$ for four different values of $\beta$. In all cases, the nonlinear drive is

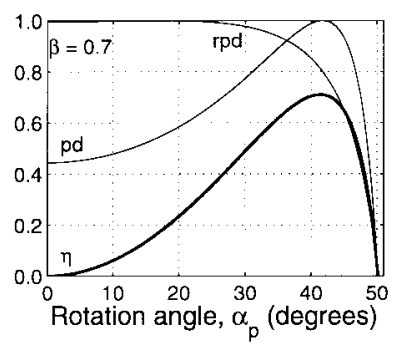

(a)

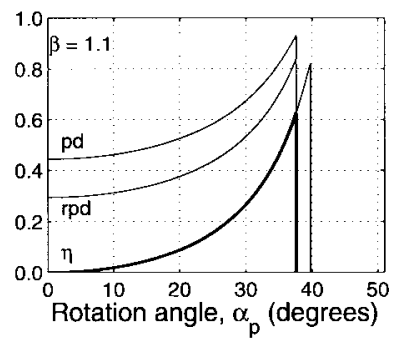

(c)

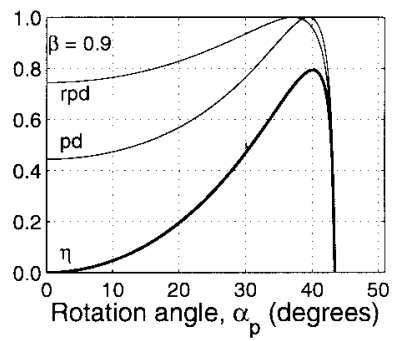

(b)

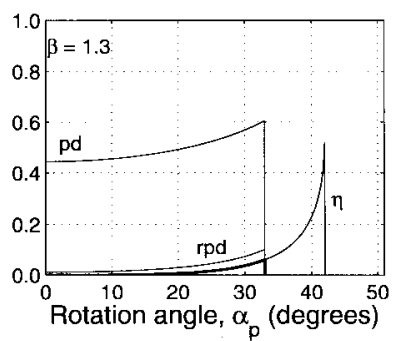

(d)
Fig. 6. Conversion efficiency $(\eta)$, pump depletion (pd), and rotated pump depletion (rpd) as functions of the pump polarization rotation angle for a class-B SF-OPO. The nonlinear drive is unity and $L=0.04$ in all cases. $\beta=$ (a) 0.7 , (b) 0.9 , (c) 1.1 , and (d) 1.3 .

kept constant at unity and $L=0.04$. At $\alpha_{p}=0^{\circ}$, the SF-OPO is above threshold but there is no SFG. As $\alpha_{p}$ is increased, the conversion efficiency increases and reaches its maximum value. In Fig. 6(a) and (b), the decrease after the peak is gradual, and the SF-OPO falls below threshold at some $\alpha_{p}$ value. However, in Fig. 6(c) and (d), the SF-OPO falls below threshold abruptly when $\alpha_{p}$ increases above its optimum value as a consequence of the threshold $\alpha_{p}$ being smaller than the optimum $\alpha_{p}$. This behavior is related to the unusual saturation characteristics of the parametric gain; starting from $g_{0}$ at $a_{2}^{2}(0)=0, g\left[a_{2}^{2}(0)\right]$ first increases with increasing $a_{2}^{2}(0)$ and then decreases to cross the loss line $1 / R$ at the intracavity signal flux density $a_{2}^{2}(0)=x_{1}$. When $\alpha_{p}$ is increased, $g_{0}$ decreases and becomes less than $1 / R$, and $a_{2}^{2}(0)=x_{0}=0$ becomes a stable fixed point. If the SF-OPO is turned on at this point, oscillations cannot build up in the cavity. However, if one increases $\alpha_{p}$ while the SF-OPO is oscillating at $a_{2}^{2}(0)=x_{1}$, the oscillations continue past the threshold $\alpha_{p}$ value, as shown in Fig. 6(c) and (d).

Both the threshold $\alpha_{p}$ and threshold nonlinear drive can be found by solving $g_{0} R=1$. Since the OPO and the SFG processes are independent in a single pass through the crystal and the retarder switches around the polarizations of $a_{2}$ and $a_{4}$ in each round trip, the signal field has to be followed for two cavity round trips to find the threshold condition. The small-signal gain over two round trips can be expressed as

$$
g_{0}=R \cosh ^{2}\left(\sqrt{D} \cos \alpha_{p}\right) \cos ^{2}\left(\beta \sqrt{D} \sin \alpha_{p}\right) .
$$

where the $\cosh ^{2}\left(\sqrt{D} \cos \alpha_{p}\right)$ factor represents the gain of the OPO signal in the first pass through the crystal, whereas the $\cos ^{2}\left(\beta \sqrt{D} \sin \alpha_{p}\right)$ factor represents the loss due to SFG in the second pass. The oscillatory nature of the $\cos ^{2}\left(\beta \sqrt{D} \sin \alpha_{p}\right)$ factor in (75) results in a number of distinct nonlinear drive ranges for which the SF-OPO is below threshold. 


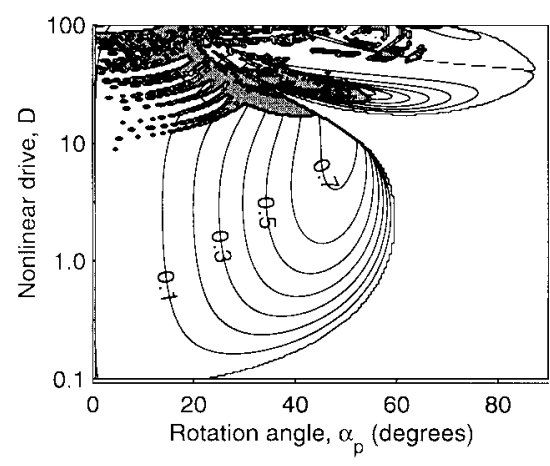

(a)

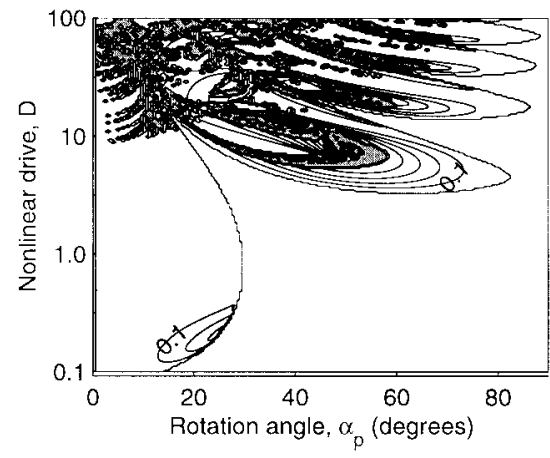

(c)

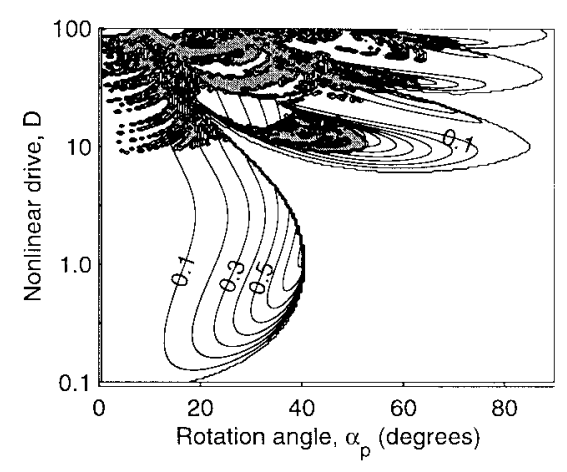

(b)

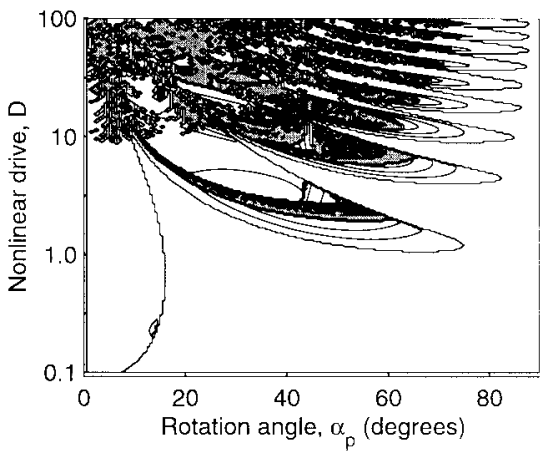

(d)

Fig. 7. Conversion efficiency as a function of the nonlinear drive and the pump polarization rotation angle for class-B SF-OPO's. (a) $\beta=0.5$. (b) $\beta=1.0$. (c) $\beta=1.5$. (d) $\beta=3.0$. For each case, $L=0.04$. All contours are in 0.1 steps.

Fig. 7 shows contour maps of the conversion efficiency as a function of the nonlinear drive and $\alpha_{p}$ for four different values of $\beta$. The contours indicate curves of equal conversion efficiency. The white area extending from the lower right corner of each plot is the region for which the SF-OPO is below threshold. The contour that borders this area is a plot of the $g_{0} R=1$ curve, representing the threshold condition. Regions of periodic and chaotic oscillations are identified with grey and black areas, respectively. In the case of a multistable steady-state solution, the figure shows the conversion efficiency value that is reached by continuously changing each parameter ( $D$ or $\alpha_{p}$ ) from a single-valued steady-state solution.

All plots in Fig. 7 show "lobes" of regions where the SF-OPO is above threshold. The number of lobes in the $D=1-100$ range increases with increasing $\beta$. The lowest lobe does not exhibit any periodic or chaotic oscillations up to approximately $D=8$, regardless of the value of $\beta$. The peak conversion efficiency in this stable lobe is highest for $\beta \approx 1$. The decline in maximum conversion efficiency is more rapid toward $\beta>1$ compared to $\beta<1$. A lower value of $\beta$ requires a higher value of nonlinear drive for optimum conversion. Therefore, in the design of a class-B SF-OPO, $\beta$ is again an important parameter. For high conversion efficiency, $\beta$ should be approximately in the $0.3-1.2$ range. To avoid periodic or chaotic oscillations, the nonlinear drive should be less than 8 .

\section{Class-C Dynamics}

The phase-matching geometry of class-C SF-OPO's leads to orthogonal polarizations for the OPO pump and the higher

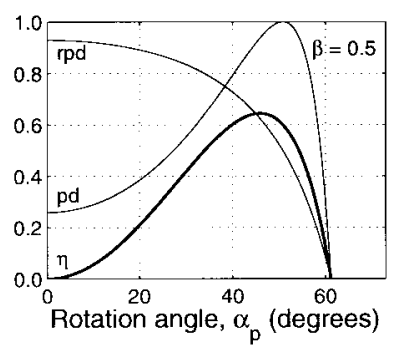

(a)

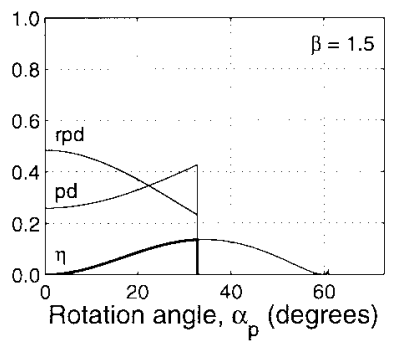

(c)

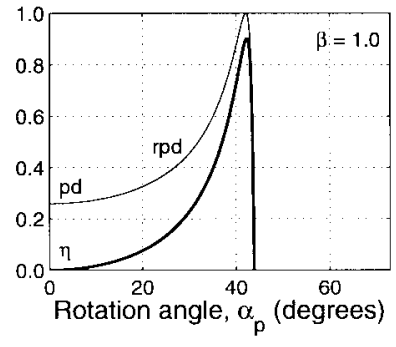

(b)

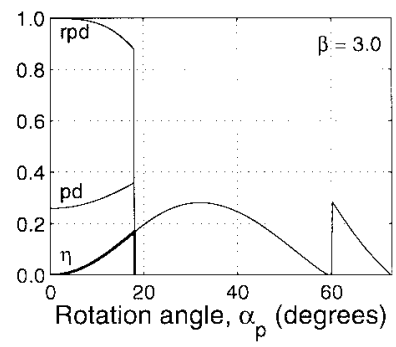

(d)
Fig. 8. Conversion efficiency $(\eta)$, pump depletion (pd), and rotated pump depletion (rpd) as functions of the pump polarization rotation angle for a class-C SF-OPO. The nonlinear drive is unity and $L=0.04$ in all cases. $\beta=$ (a) 0.5 , (b) 1.0 , (c) 1.5 , and (d) 3.0 .

frequency SFG input fields. A half-wave retarder provides adjustable rotation of the pump polarization before the pump beam enters the cavity. For a polarization rotation angle of $\alpha_{p}$, a $\sin ^{2} \alpha_{p}$ fraction of the total input pump flux density $a_{p}^{2}$ is used as the higher frequency SFG input $a_{5}^{2}(0)$, whereas the remaining pump photon flux density $a_{3}^{2}(0)$ provides parametric 


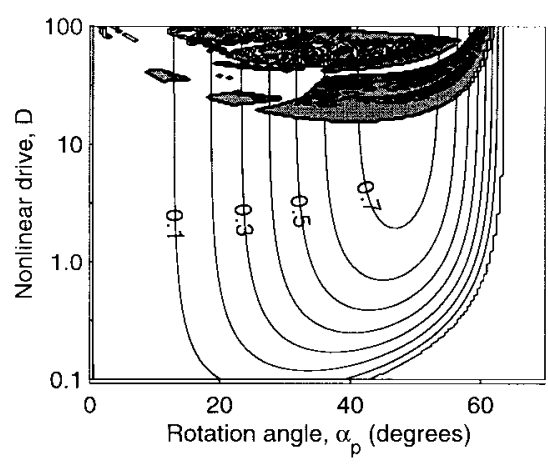

(a)

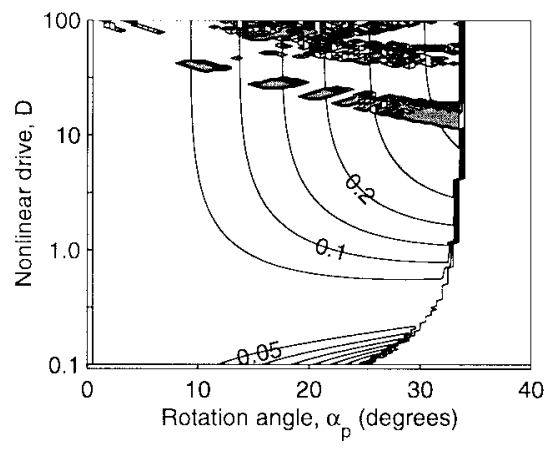

(c)

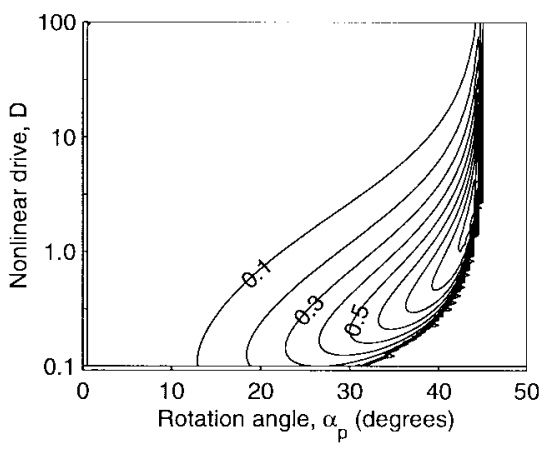

(b)

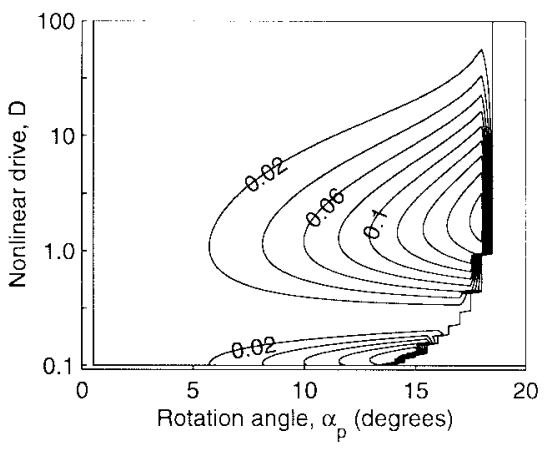

(d)

Fig. 9. Conversion efficiency as a function of the nonlinear drive and the pump polarization rotation angle for class-C SF-OPO's. $\beta=$ (a) 0.5 , (b) 1.0 , (c) 1.5, and (d) 3.0. For each case, $L=0.04$.

gain for the signal field. With this configuration, the distribution of the pump photon flux between the two processes can be adjusted to maximize the conversion efficiency.

As an example, Fig. 8 shows the conversion efficiency and the depletion of the pump and the rotated pump (higher frequency SFG input) fields as functions of the polarization rotation angle $\alpha_{p}$ for four different values of $\beta$, where the nonlinear drive is kept constant at unity and $L=0.04$.

For $\beta=0.5$ in Fig. 8(a), maximum conversion efficiency and full pump depletion occur at different values of $\alpha_{p}$. The depletion of the rotated pump decreases monotonically until the SF-OPO gets below threshold. For $\beta=1$ in Fig. 8(b), complete pump depletion coincides with maximum conversion. For this particular value of $\beta$, the depletion of the pump and the rotated pump fields are equal for all values of $\alpha_{p}$ and $D$, since $\gamma(z)=\theta(z)$ for $\beta=1$. Simultaneous depletion of both pump components is possible only when $\beta$ or $1 / \beta$ is an odd integer, since $\cos (\beta \theta)$ and $\cos \theta$ can become equal to zero at the same $\theta$ value only then. For $\beta$ larger than unity, the conversion efficiency achieves its maximum right before the threshold $\alpha_{p}$, similar to class-B SF-OPO's. Fig. 8(c) and (d) show examples of this behavior for $\beta=1.5$ and 3 , respectively. Note that a stable nonzero fixed point of (69) may exist beyond the threshold $\alpha_{p}$, even though iterations starting out from near zero cannot reach this fixed point.

Both the threshold nonlinear drive and the threshold $\alpha_{p}$ can be found by equating the small-signal gain

$$
g_{0}=\cosh ^{2}\left(\cos \alpha_{p} \sqrt{\left(1-\beta^{2} \tan ^{2} \alpha_{p}\right) D}\right)
$$

to $1 / R$. Note that (76) is valid only if $\beta \tan \alpha_{p} \leq 1$, otherwise $g_{0}<1$.

Fig. 9 shows contour maps of the conversion efficiency as a function of the nonlinear drive and $\alpha_{p}$ for four different values of $\beta$. In Fig. 9(a) and (c), periodic and chaotic oscillations occur approximately for $D>10$. However, if $\beta$ has an integer value as in Fig. 9(b) and (d), there are no periodic or chaotic oscillations. As $\beta$ approaches an integer value, the onset of periodic and chaotic oscillations move out to higher values on the nonlinear drive, reaching infinite nonlinear drive at integer $\beta$ values. Multistability is encountered at high nonlinear drive values regardless of the value of $\beta$. Note that, for $\beta>1$, there are "valleys" of very low conversion efficiency, some even at high nonlinear drive values. In the design of class-C SFOPO's, $\beta$ is again an important parameter. For high conversion efficiency, $\beta$ should be approximately in the $0.3-1.2$ range.

\section{Class-D Dynamics}

In class-D SF-OPO's, the polarizations of the OPO signal $a_{2}$ and the lower frequency SFG input $a_{4}$ are orthogonal. An intracavity half-wave retarder provides the SFG input $a_{4}(0)$ by rotating the signal polarization. There are two modes of operation, as in class-B SF-OPO's, depending on the signal polarization rotation angle $\alpha_{s}$.

If $\alpha_{s}$ is set to $90^{\circ}$, the retarder switches around the polarizations of the OPO signal $a_{2}(l)$ and the lower frequency SFG field $a_{4}(l)$ after each pass through the crystal. Fig. 10 shows the conversion efficiency as a function of the nonlinear drive for four different values of $\beta$. For $\beta=0.5$, the SF- 


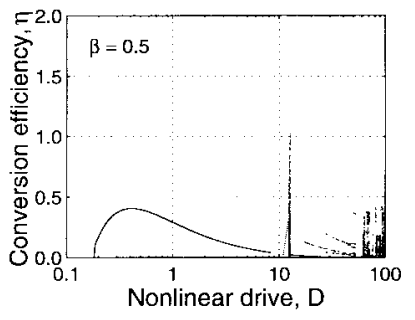

(a)

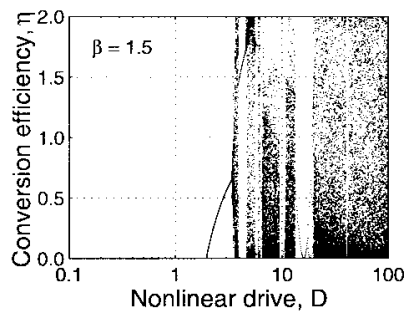

(c)

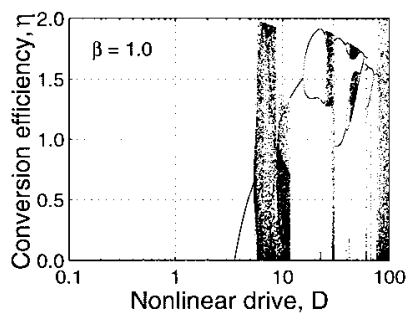

(b)

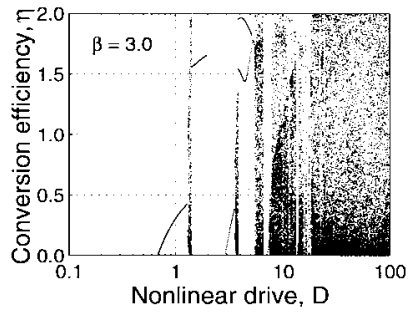

(d)

Fig. 10. Conversion efficiency as a function of the nonlinear drive for various values of $\beta$ for a class-D SF-OPO with a signal polarization rotation angle of $90^{\circ} \cdot \beta=$ (a) 0.5 , (b) 1.0 , (c) 1.5 , and (d) 3.0. For each case, $L=0.04$.

OPO has a broad region of steady-state operation with a peak conversion efficiency of 0.40 at a nonlinear drive of 0.41 . Chaotic behavior is observed at larger values of the nonlinear drive. For larger $\beta$ values, the regions of steady-state operation are narrower and the SF-OPO exhibits chaotic oscillations for most nonlinear drive values. Some of the chaotic regions begin abruptly without any period doubling, and at relatively low values of the nonlinear drive when compared to other classes.

For the configuration where $0^{\circ}<\alpha_{s}<90^{\circ}$, we assume that the rotated signal field (lower frequency SFG input) at the output of the crystal is either coupled out of the cavity with a polarizing beamsplitter or strongly depleted, so that interference due to polarization mixing at the half-wave retarder is avoided. In this configuration, maximum conversion to the sum-frequency takes place when both the pump and the rotated signal fields are fully depleted at the output. The nonlinear drive and $\alpha_{s}$ can be adjusted to deplete these two fields completely and maximize $\eta$.

The depletion of the rotated signal does not depend on the value of the nonlinear drive. To arrive at this conclusion, we first note that $R a_{2}^{2}(l)=a_{2}^{2}(0)+a_{4}^{2}(0)$, and obtain

$$
\frac{a_{2}^{2}(l)}{a_{2}^{2}(0)}=\cosh ^{2} \theta(l)=\frac{1+\tan ^{2} \alpha_{s}}{R}
$$

where $\theta(z)$ is the solution to (66). This relation shows that $\theta(l)$ depends only on $\alpha_{s}$ and $R$. The rotated signal depletion at the output is

$$
1-\frac{a_{4}^{2}(l)}{a_{4}^{2}(0)}=1-\cos ^{2}(\beta \theta(l))
$$

and independent of the value of the nonlinear drive.

For the rotated signal to be fully depleted, the product $\beta \theta(l)$ should be equal to an odd multiple of $\pi / 2$. Since the $\beta \theta(l)=\pi / 2$ case requires smaller values for the nonlinear drive and $\alpha_{s}$, we consider this case only. Using (77), the angle

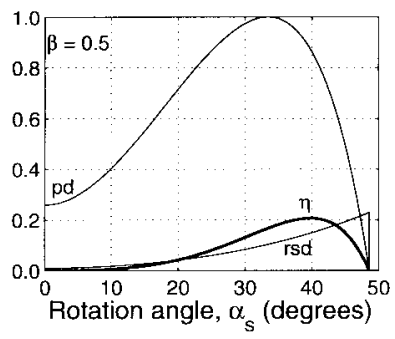

(a)

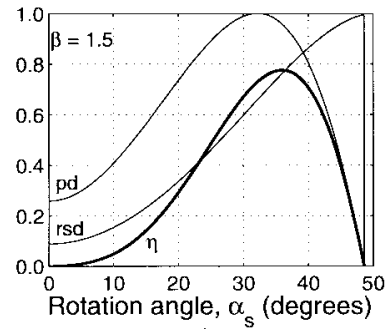

(c)

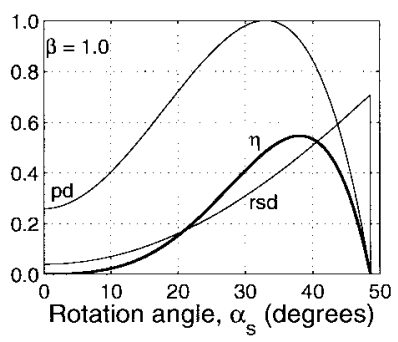

(b)

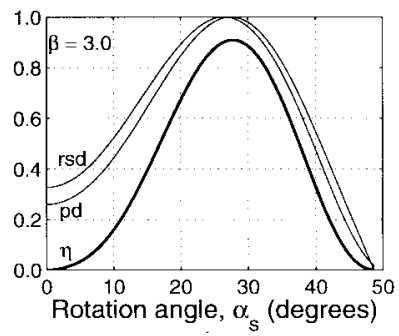

(d)
Fig. 11. Conversion efficiency $(\eta)$, pump depletion (pd), and rotated signal depletion (rsd) as functions of the signal polarization rotation angle for a class-D SF-OPO. $\beta=$ (a) 0.5 , (b) 1.0, (c) 1.5, and (d) 3.0. The nonlinear drive is unity and $L=0.04$ in all cases.

$\alpha_{s}$ that depletes the rotated signal for given values of $\beta$ and $R$ is found to be

$$
\alpha_{s}=\tan ^{-1} \sqrt{R \cosh ^{2}(\pi / 2 \beta)-1}
$$

Complete depletion of the pump takes place when the first term in (66) representing the pump photon flux density is equal to zero. This condition allows us to obtain the ratio of $C_{1}$ and $C_{2}$ as

$$
m=\frac{C_{1}}{C_{2}}=\frac{1}{\cosh ^{2} \theta+\tan ^{2} \alpha_{s} \sin ^{2}(\beta \theta)} .
$$

This relation also determines the ratio of the intracavity signal flux to the input pump flux through the Manley-Rowe relations (62) and (63). In the case of simultaneous depletion of the pump and the rotated signal fields at the crystal output, (80) can be expressed in terms of $\beta$ and $R$ as

$$
m_{\mathrm{opt}}=\frac{1}{(R+1) \cosh ^{2}(\pi / 2 \beta)-1} .
$$

We then integrate (66) and obtain the optimum nonlinear drive as

$$
\begin{aligned}
D_{\mathrm{opt}}= & \left(1-m_{\mathrm{opt}}\right)\left[\int _ { 0 } ^ { \pi / 2 \beta } \left[1-m_{\mathrm{opt}}\left(\cosh ^{2} \phi\right.\right.\right. \\
& \left.\left.\left.+\tan ^{2} \alpha_{s} \sin ^{2}(\beta \phi)\right)\right]^{-1 / 2} d \phi\right]^{2}
\end{aligned}
$$

where $\alpha_{s}$ and $m_{\mathrm{opt}}$ are given by (79) and (81), respectively. The integral in (82) has an integrable singularity at its upper limit and can be evaluated numerically after a change of variable $\phi=\pi / 2 \beta-\rho^{2}$ eliminates the singularity [31].

The optimum nonlinear drive $D_{\mathrm{opt}}$ decreases with increasing values of $\beta$. The maximum conversion efficiency achieved 


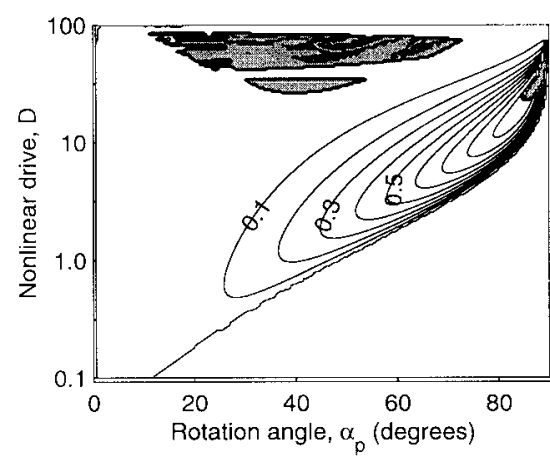

(a)

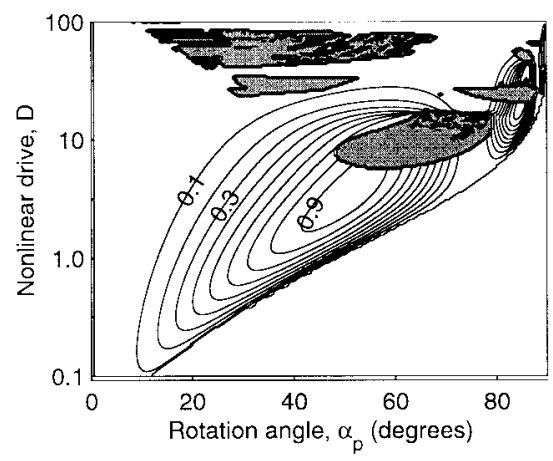

(c)

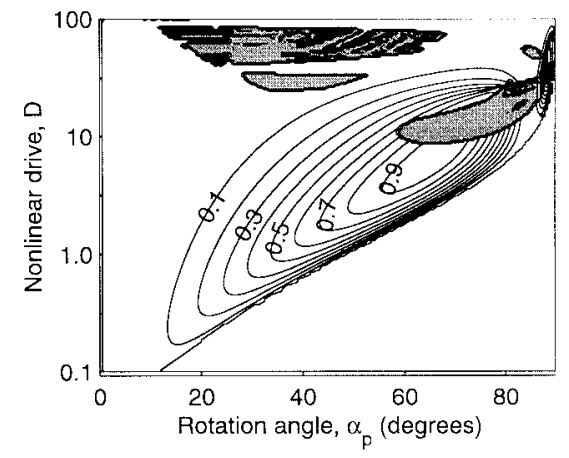

(b)

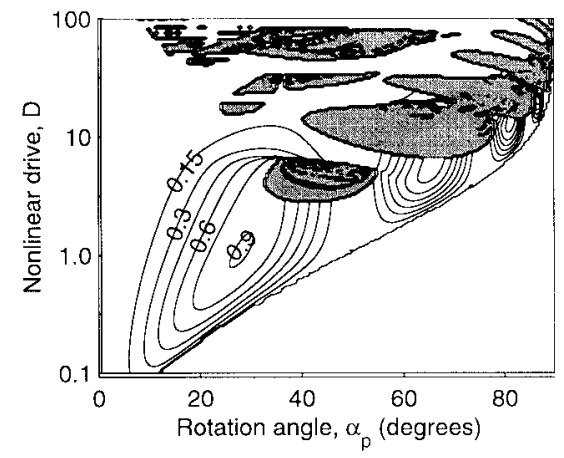

(d)

Fig. 12. Conversion efficiency as a function of the nonlinear drive and the pump polarization rotation angle for class-D SF-OPO's. $\beta=$ (a) 0.5 , (b) 1.0 , (c) 1.5 , and (d) 3.0. For each case, $L=0.04$.

at $D_{\mathrm{opt}}$ is

$$
\eta_{\max }=2 \frac{R \cosh ^{2}(\pi / 2 \beta)-1}{(R+1) \cosh ^{2}(\pi / 2 \beta)-2} .
$$

For larger values of $\beta$, the maximum conversion efficiency decreases rapidly with increasing cavity losses.

As an example, Fig. 11 shows $\eta$ and the depletion of the pump and the rotated signal flux densities as functions of $\alpha_{s}$ for four different values of $\beta$, where $D$ is kept constant at unity and $L=0.04$. Note that, as $\beta$ gets larger, the peak conversion efficiency increases and shifts to smaller values of $\alpha_{s}$. The threshold $\alpha_{s}$ is independent of $\beta$ since the net small-signal gain $g_{0}=\cos ^{2} \alpha_{s} \cosh ^{2} \sqrt{D}$, which includes the linear loss due to polarization rotation, is not dependent on $\beta$.

Fig. 12 shows contour maps of the conversion efficiency as a function of the nonlinear drive and $\alpha_{p}$ for four different values of $\beta$. Highly efficient conversion is possible regardless of the value of $\beta$. As $\beta$ get larger, the location of the peak conversion efficiency shifts to smaller values of the nonlinear drive, together with the onset of periodic and chaotic oscillations. In the design of class-D SF-OPO's, $\beta$ should be chosen with the available nonlinear drive in mind.

\section{CONCLUSION}

The simultaneous phase matching of two different secondorder nonlinear processes within the same crystal with one or more fields in common gives rise to a wide range of frequencyconversion applications. Both birefringent and quasi-phasematching techniques can be used to phase match either process. Quasi-phase matching offers added flexibility in the choice of wavelengths and brings adjustability to the relative strength of the two processes.

In this paper, we investigated upconversion OPO's based on the simultaneous phase matching of parametric generation and SFG, where the resonant OPO signal is summed with a portion of the pump. We have identified four distinct classes of SF-OPO's depending on which field components are common. These four classes are characterized by different sets of coupled mode equations and, consequently, show different characteristics from each other. However, efficient upconversion is possible in all four classes with careful design. The most important design parameters are the nonlinear drive and $\beta$. For a desired set of wavelengths, the nonlinear drive depends on the effective nonlinear coefficient of the crystal $d_{e}$, the crystal length $l$, and the available pump photon flux density $a_{p}^{2}$. Even though the pump flux density can be adjusted by changing the beam size, experimental constraints such as keeping the confocal distance longer than the crystal length or crystal damage threshold may limit the maximum available $a_{p}^{2}$. The useful crystal length is also limited; physical limitations arise from crystal growth constraints, or in pulsed systems, the pulse overlap length may be limited due to group velocity mismatch between the field components. As a result, there is usually an upper limit to the available nonlinear drive. The parameter $\beta$ is more difficult to tailor. Once the phasematched wavelengths are known, BPM offers no method for adjusting $\beta$. However, using QPM for either one or both processes provides a mechanism for adjusting $\beta$. The polarization rotation required in classes $\mathrm{B}, \mathrm{C}$, and $\mathrm{D}$ provides an adjustable parameter, the polarization rotation angle $\alpha$, that 
may be easily changed by rotating a half-wave retarder to maximize the conversion efficiency. The cavity losses $L$ also influence the performance of SF-OPO's. In all classes except class A, it is best to minimize the cavity losses; in a typical experiment, one can usually keep $L<0.05$. For class A, however, one of the cavity mirrors may have to be replaced with an output coupler for the signal field in order to maximize the conversion efficiency.

In class-A SF-OPO's, the maximum possible conversion efficiency is entirely determined by $\beta$. The conversion efficiency increases monotonically with increasing $\beta$ for values less than unity. However, the SF-OPO cannot get above threshold for $\beta \geq 1$. Therefore, it is desirable to have $\beta$ to be as high as possible without exceeding unity. Once $\beta$ is fixed, the maximum possible conversion efficiency is known. The nonlinear drive at which this efficiency can be realized depends on the cavity losses. If the available nonlinear drive is high enough, one can increase the cavity losses with an output coupling mirror to achieve the maximum possible efficiency, or otherwise keep the cavity losses as low as possible.

In class-B SF-OPO's, the polarizations of the signal and rotated signal fields are switched around at each pass, while the pump polarization angle $\alpha_{p}$ is continuously adjustable. For a given nonlinear drive and $\beta$, one can maximize the conversion efficiency by adjusting $\alpha_{p}$. However, this maximum conversion efficiency is a strong function of $\beta$. As $\beta$ increases from zero, the efficiency increases, reaching a peak close to unity conversion around $\beta \simeq 1 \pm 0.3$, the exact values depending on the nonlinear drive. With a further increase in $\beta$, the efficiency decreases rapidly. On the other hand, when $\alpha_{p}$ is increased above a certain threshold value, the small-signal gain becomes too small to compensate for the cavity losses $\left(g_{0}<1 / R\right)$. However, efficient conversion may be possible even above this threshold $\alpha_{p}$, if the SF-OPO is turned on at a lower $\alpha_{p}$ where $g_{0} R>1$, and then $\alpha_{p}$ is gradually increased past its threshold value.

In class-C SF-OPO's, the pump polarization angle $\alpha_{p}$ is continuously adjustable. The SF-OPO can get above threshold for any value of $\beta$. Again, for a given nonlinear drive and $\beta$, one can maximize the conversion efficiency by adjusting $\alpha_{p}$. The $\beta$ dependence of the maximum efficiency shows a pattern similar to the class-B case. Efficient operation past the threshold $\alpha_{p}$ is also possible as in class-B SF-OPO's.

In class-D SF-OPO's, the intracavity signal polarization angle $\alpha_{s}$ is continuously adjustable. For a given nonlinear drive and $\beta$, one can maximize the conversion efficiency by adjusting $\alpha_{s}$. As $\beta$ increases from zero, the efficiency increases, reaching a peak close to unity conversion around $\beta \simeq 1$, as in classes $\mathrm{B}$ and $\mathrm{C}$, but stays high with a further increase in $\beta$.

Multistable operation is encountered in all classes at relatively high nonlinear drive values. Here, the SF-OPO may exhibit one of a number of conversion efficiency values depending on the initial signal flux density. It may be possible to force the SF-OPO to one of the stable states by seeding the oscillator.

Period doubling and chaotic behavior is observed in all classes except class A at relatively high values of the non- linear drive. In these situations, the instantaneous conversion efficiency may be higher than unity.

We conclude that SF-OPO's are promising devices for the efficient upconversion of lasers. The development of accurate computational models that take the transverse and temporal variations of the beams into account would be very useful in the practical design and optimization of SF-OPO's.

\section{REFERENCES}

[1] Special issue, "Optical parametric oscillation and amplification," J. Opt. Soc. Amer. B, vol. 10, pp. 1659-2243, 1993.

[2] Special issue, "Optical parametric devices," J. Opt. Soc. Amer. B, vol. 12, pp. 2087-2320, 1995.

[3] C. L. Tang, W. R. Bosenberg, T. Ukachi, R. J. Lane, and L. K. Cheng, "Optical parametric oscillators," Proc. IEEE, vol. 80, pp. 365-374, 1992

[4] R. J. Ellingson and C. L. Tang, "High-power, high-repetition-rate femtosecond pulses tunable in the visible," Opt. Lett., vol. 18, pp. 438-440, 1993

[5] E. C. Cheung, K. Koch, and G. T. Moore, "Frequency upconversion by phase-matched sum-frequency generation in an optical parametric oscillator," Opt. Lett., vol. 19, pp. 1967-1969, 1994.

[6] P. P. Bey and C. L. Tang, "Plane-wave theory of parametric oscillator and coupled oscillator-upconverter," IEEE J. Quantum Electron., vol. QE-8, pp. 361-369, 1972.

[7] G. T. Moore, K. Koch, and E. C. Cheung, "Optical parametric oscillation with intracavity second-harmonic generation," Opt. Commun., vol. 113, pp. 463-470, 1995

[8] G. T. Moore and K. Koch, "Optical parametric oscillation with intracavity sum-frequency generation," IEEE J. Quantum Electron., vol. 29 , pp. 961-969, 1993.

[9] T. Kartaloğlu, K. G. Köprülü, and O. Aytür, "Phase-matched selfdoubling optical parametric oscillator," Opt. Lett., vol. 22, pp. 280-282, 1997.

[10] K. G. Köprülü, T. Kartaloğlu, and O. Aytür, in Conf. Lasers and ElectroOptics, OSA Tech. Dig. Series, 1997, vol. 11, p. 457.

[11] R. A. Andrews, H. Rabin, and C. L. Tang, "Coupled parametric downconversion and upconversion with simultaneous phase matching," Phys. Rev. Lett., vol. 25, pp. 605-608, 1970.

[12] J. M. Yarborough and E. O. Ammann, "Simultaneous optical parametric oscillation, second harmonic generation, and difference frequency generation," Appl. Phys. Lett., vol. 18, pp. 145-147, 1970.

[13] C. McGowan, D. T. Reid, Z. E. Penman, M. Ebrahimzadeh, W. Sibbett, and D. H. Jundt, "Femtosecond optical parametric oscillator based on periodically poled lithium niobate," J. Opt. Soc. Amer. B, vol. 15, pp. 694-701, 1998

[14] D. T. Reid, G. T. Kennedy, A. Miller, W. Sibbett, and M. Ebrahimzadeh, "Widely tunable, near- to mid-infrared femtosecond and picosecond optical parametric oscillators using periodically poled $\mathrm{LiNbO}_{3}$ and $\mathrm{RbTiOAsO}_{4}$," IEEE J. Select. Topics Quantum Electron., vol. 4, pp. 238-248, 1998

[15] K. C. Burr, C. L. Tang, M. A. Arbore, and M. M. Fejer, "Broadly tunable mid-infrared femtosecond optical parametric oscillator using all-solidstate-pumped periodically poled lithium niobate," Opt. Lett., vol. 22, pp. 1458-1460, 1997.

[16] S. D. Butterworth, P. G. R. Smith, and D. C. Hanna, "Picosecond Ti:Sapphire-pumped optical parametric oscillator based on periodically poled $\mathrm{LiNbO}_{3}$," Opt. Lett., vol. 22, pp. 618-620, 1997.

[17] M. Vaidyanathan, R. C. Eckardt, V. Dominic, L. E. Myers, and T. P. Grayson, "Cascaded optical parametric oscillations," Opt. Express, vol. 1, pp. 49-53, 1997.

[18] V. Petrov and F. Noack, "Frequency upconversion of tunable femtosecond pulses by parametric amplification and sum-frequency generation in a single nonlinear crystal," Opt. Lett., vol. 20, pp. 2171-2173, 1995.

[19] O. Pfister, J. S. Wells, L. Hollberg, L. Zink, D. A. Van Baak, M. D. Levenson, and W. R. Bosenberg, "Continuous-wave frequency tripling and quadrupling by simultaneous three-wave mixings in periodically poled crystals: Application to a two-step 1.19-10.71- $\mu \mathrm{m}$ frequency bridge," Opt. Lett., vol. 22, pp. 1211-1213, 1997.

[20] O. Aytür and Y. Dikmelik, "Plane-wave theory of self-doubling optical parametric oscillators," IEEE J. Quantum Electron., vol. 34, pp. 447-458, 1998.

[21] R. A. Baumgartner and R. L. Byer, "Optical parametric amplification," IEEE J. Quantum Electron., vol. QE-15, pp. 432-444, 1979.

[22] R. W. Boyd, Nonlinear Optics. San Diego, CA: Academic, 1992. 
[23] M. Abramowitz and I. A. Stegun, Eds., Handbook of Mathematical Functions with Formulas, Graphs, and Mathematical Tables. New York: Dover, 1965

[24] D. A. Roberts, "Simplified characterization of uniaxial and biaxial nonlinear optical crystals," IEEE J. Quantum Electron., vol. 28, pp. 2057-2074, 1992

[25] L. E. Myers, R. C. Eckardt, M. M. Fejer, R. L. Byer, W. R. Bosenberg, and J. W. Pierce, "Quasi-phase-matched optical parametric oscillators in bulk periodically poled $\mathrm{LiNbO}_{3}$," J. Opt. Soc. Amer. B, vol. 12, pp. 2102-2116, 1995.

[26] W. R. Bosenberg, J. I. Alexander, L. E. Myers, and R. W. Wallace, "2.5-W, continuous-wave, 629-nm solid-state laser source," Opt. Lett., vol. 23, pp. 207-209, 1998.

[27] R. L. Burden and J. D. Faires, Numerical Analysis, 5th ed. Boston, MA: PWS Publishing, 1993.

[28] R. L. Devaney, An Introduction to Chaotic Dynamical Systems, 2nd ed. Redwood City, CA: Addison-Wesley, 1989.

[29] R. A. Holmgren, A First Course in Discrete Dynamical Systems, 2nd ed. New York: Springer, 1996.

[30] R. L. Byer and S. E. Harris, "Power and bandwidth of spontaneous parametric emission," Phys. Rev., vol. 168, pp. 1064-1068, 1968.

[31] W. H. Press, S. A. Teukolsky, W. T. Vetterling, and B. P. Flannery, Numerical Recipes in $C$, 2nd ed. Cambridge. U.K.: Cambridge Univ., 1992.

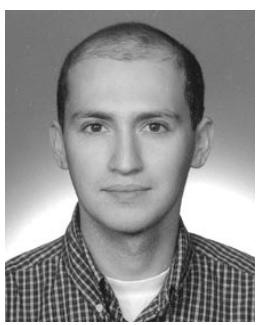

Yamaç Dikmelik (S'93) was born in Izmir, Turkey, in 1974. He received the B.S. and M.S. degrees in electrical engineering from Bilkent University, Ankara, Turkey, in 1996 and 1998, respectively. He is presently a graduate student at The Johns Hopkins University, Baltimore, MD.
Gülbin Akgün was born in Izmir, Turkey, in 1974. She received the B.S and M.S. degrees in electrical engineering from Bilkent University, Ankara, Turkey, in 1996 and 1999, respectively.

She is presently with Mikes Corporation, Ankara, Turkey.

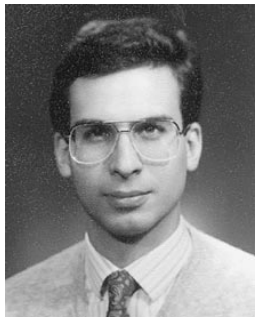

Orhan Aytür (M'94) was born in Ankara, Turkey, in 1965 . He received the B.S. degree in electrica engineering from Middle East Technical University, Ankara, Turkey, in 1986, and the M.S. and Ph.D degrees in electrical engineering from Northwestern University, Evanston, IL, in 1988 and 1991, respectively.

He worked as a Laser Scientist at Fibertek, Inc., in Virginia, and as a Research Associate at the University of New Mexico, Albuquerque, during 1991-1992. He joined the Department of Electrical Engineering at Bilkent University in Ankara, Turkey, in 1993, where he is presently an Associate Professor. His main research interests include nonlinear frequency conversion of lasers, quantum optics, and high-performance photodetectors. 